\title{
Barriers and facilitators to implementing evidence-based guidelines in long-term care: a qualitative evidence synthesis
}

Caitlin McArthur ${ }^{1,2^{*}}$ D, Yuxin Bai ${ }^{2,3}$, Patricia Hewston ${ }^{2,3}$, Lora Giangregorio ${ }^{4,5}$, Sharon Straus ${ }^{6}$ and Alexandra Papaioannou ${ }^{2,3}$

\begin{abstract}
Background: The long-term care setting poses unique challenges and opportunities for effective knowledge translation. The objectives of this review are to (1) synthesize barriers and facilitators to implementing evidencebased guidelines in long-term care, as defined as a home where residents require 24-h nursing care, and 50\% of the population is over the age of 65 years; and (2) map barriers and facilitators to the Behaviour Change Wheel framework to inform theory-guided knowledge translation strategies.

Methods: Following the guidance of the Cochrane Qualitative and Implementation Methods Group Guidance Series and the ENTREQ reporting guidelines, we systematically reviewed the reported experiences of long-term care staff on implementing evidence-based guidelines into practice. MEDLINE Pubmed, EMBASE Ovid, and CINAHL were searched from the earliest date available until May 2021. Two independent reviewers selected primary studies for inclusion if they were conducted in long-term care and reported the perspective or experiences of long-term care staff with implementing an evidence-based practice guideline about health conditions. Appraisal of the included studies was conducted using the Critical Appraisal Skills Programme Checklist and confidence in the findings with the GRADE-CERQual approach.

Findings: After screening 2680 abstracts, we retrieved 115 full-text articles; 33 of these articles met the inclusion criteria. Barriers included time constraints and inadequate staffing, cost and lack of resources, and lack of teamwork and organizational support. Facilitators included leadership and champions, well-designed strategies, protocols, and resources, and adequate services, resources, and time. The most frequent Behaviour Change Wheel components were physical and social opportunity and psychological capability. We concluded moderate or high confidence in all but one of our review findings.
\end{abstract}

Conclusions: Future knowledge translation strategies to implement guidelines in long-term care should target physical and social opportunity and psychological capability, and include interventions such as environmental restructuring, training, and education.

Keywords: Long-term care, Barriers, Facilitators, Evidence-based, Guidelines, Knowledge translation

\footnotetext{
* Correspondence: caitlin.mcarthur@dal.ca

'Dalhousie University, 5869 University Avenue, Halifax, Nova Scotia B3H 4R2,

Canada

${ }^{2}$ GERAS Centre for Aging Research, 88 Maplewood Avenue, Hamilton,

Ontario L8M 1W9, Canada

Full list of author information is available at the end of the article
}

C C The Author(s). 2021 Open Access This article is licensed under a Creative Commons Attribution 4.0 International License, which permits use, sharing, adaptation, distribution and reproduction in any medium or format, as long as you give appropriate credit to the original author(s) and the source, provide a link to the Creative Commons licence, and indicate if changes were made. The images or other third party material in this article are included in the article's Creative Commons licence, unless indicated otherwise in a credit line to the material. If material is not included in the article's Creative Commons licence and your intended use is not permitted by statutory regulation or exceeds the permitted use, you will need to obtain permission directly from the copyright holder. To view a copy of this licence, visit http://creativecommons.org/licenses/by/4.0/. The Creative Commons Public Domain Dedication waiver (http://creativecommons.org/publicdomain/zero/1.0/) applies to the data made available in this article, unless otherwise stated in a credit line to the data. 


\section{Contributions to the literature}

- Evidence-based guidelines enhance the provision of care. However, trial-and-error-based approaches to implementation are costly and ineffective.

- This review summarizes knowledge on contextual factors in the long-term care setting that influence implementation of evidence-based guidelines to facilitate more effective and sustainable uptake in practice.

- By placing the findings of our qualitative evidence synthesis within the context of a behaviour change framework, our work provides theory-guided strategies to inform future translation of evidence into practice in long-term care homes.

\section{Background}

\section{Description of the topic}

Evidence-based guidelines summarize the best available research on health care practices to enhance the provision of consistent and appropriate care [1]. However, bringing evidence into clinical practice is an ongoing challenge. Systematic reviews on guideline adherence and utilization found that a large percentage of available guidelines do not have sustained implementation where appropriate [2, 3]. For example, an organization may implement a new guideline into practice, but the behaviours associated with it do not continue after initial introduction. In contrast, if new evidence emerges, suggesting current practices are not effective, they must be de-adopted. Guideline implementation into routine healthcare can be unpredictable, and trial-and-error approaches have been costly and ineffective, producing variable results of guideline dissemination and implementation $[4,5]$. Consequently, there has been increasing interest in employing theories, models, and frameworks to direct guideline implementation. Knowledge translation focuses on developing ways to efficiently and effectively translate evidence-based knowledge into clinical care. Theory-based guideline implementation is desirable as it ensures the implementation plan and processes consider complex factors that influence success of guideline uptake prior to implementation. In this way, implementers navigate around potential pitfalls to successful implementation by conscientiously accounting for previously identified factors which could hinder their success.

Many existing knowledge translation frameworks guide researchers to consider complex factors that influence the success of guideline uptake prior to the implementation process [6-8]. The Behaviour Change Wheel is one framework that prompts users to select knowledge translation interventions based on physical, social, psychological, and environmental factors that influence the capability, opportunity, and motivation needed for behaviour change (COM-B) [7]. Central to the Behaviour Change Wheel, the COM-B system incorporates Capability, Opportunity, and Motivation as sources of Behaviour. Users can determine what needs to change for the desired behaviour (e.g., guideline implementation) to occur by identifying barriers and facilitators and mapping them onto the COM-B system. The Behaviour Change Wheel then guides users to select potential knowledge translation interventions based on their COM-B analysis [7]. Therefore, by studying barriers and facilitators in a context-specific environment, interventions can be designed in a theory-informed manner which increases the potential for sustainable practice change.

\section{Why is it important to do this review?}

The need to effectively translate evidence-based guidelines into practice is especially pressing for older adults [9] as the proportion of the population aged 65 years and over is growing exponentially [10]. Older adults with complex needs and comorbidities often live in long-term care (LTC) homes, which are living spaces for adults who have significant health challenges to receive access to 24-h nursing and personal care [11]. Guidelines have been developed for various health conditions in LTC homes ranging from diabetes to pressure ulcer prevention [12]. However, most knowledge translation studies on guideline implementation for older adults do not include LTC homes [13]. Knowledge translation strategies from other settings are poorly transferable to LTC because of the skill mix of the staff, environment, complexity of the residents' conditions, and availability of resources [14]. Knowledge translation strategies must be specifically designed for LTC given the unique context of health care provision in this setting. While barriers and facilitators to guideline implementation have been systematically reviewed in other healthcare settings [13, 15], no such analyses have been conducted for the LTC sector.

\section{How this review might inform what is already known in this area}

The findings of our study will synthesize barriers and facilitators to evidence-based guideline implementation across health conditions in LTC and mapped onto the COM-B components. Our identified barriers and facilitators and suggested knowledge translation strategies based on the COM-B mapping can be used to design theory-guided knowledge translation interventions in LTC. This will save time, effort, and resources in identifying barriers and facilitators so that planners can design interventions more quickly and efficiently. Further, our review will identify gaps in research related to evidence- 
based guideline implementation in LTC and make suggestions for future work.

\section{Objectives}

The objectives of this qualitative evidence synthesis are to (1) synthesize barriers and facilitators that LTC staff experience during the implementation of evidence-based guidelines and (2) map the identified barriers and facilitators to the central component of the Behaviour Change Wheel framework to inform future theoryguided knowledge translation intervention development in the LTC setting. Our research question is "What are the barriers and facilitators to implementing evidencebased health care guidelines in LTC homes from the perspectives of staff (e.g., nurses, health care aides, physicians)?" The phenomena of interest is implementation of health care guidelines into practice and the factors that hinder or facilitate implementation. The context is LTC homes who provide 24-h nursing care for mostly frail, medically complex older adults across the world in the 21st century.

\section{Methods}

We conducted a qualitative evidence synthesis following the guidance of the Cochrane Qualitative and Implementation Methods Group Guidance Series [16] and the ENTREQ reporting guidelines (Checklist can be found in Additional file 1) [17].

\section{Criteria for considering studies for this review Types of studies}

We included primary studies that use qualitative study designs such as ethnography, phenomenology, case studies, grounded theory studies, and qualitative process evaluations. We included studies that use both qualitative methods for data collection (e.g., focus group discussions, individual interviews, observation, diaries, document analysis, open-ended survey questions) and qualitative methods for data analysis (e.g., thematic analysis, framework analysis, grounded theory). We included studies that collect data using qualitative methods but do not analyse these data using qualitative analysis methods (e.g., open-ended survey questions where the response data are analysed using descriptive statistics only) as long as the results or findings identify barriers and facilitators as described below. We only included published studies written in English. We did not exclude studies based on our assessment of methodological limitations. We used this information about methodological limitations to assess our confidence in the review findings.

\section{Target behaviour}

The target behaviour was implementing evidence-based guidelines into practice (e.g., pressure injury management, pain, fractures, deprescribing). Barriers were defined as any factors that obstruct the capacity for LTC staff and homes to implement guidelines, while facilitators were any factors that enable implementation.

\section{Participants}

The group required to perform the target behaviour was LTC staff which included personal support workers, clinicians (e.g., nurses, physicians, pharmacists, dieticians, physiotherapists), and home administration (e.g., directors of care).

\section{Setting}

Studies were included if they were conducted in LTC, defined as a home where residents require 24-h nursing care, and $50 \%$ of the population is over the age of 65 years.

\section{Search methods for identification of studies}

Relevant articles were identified through a pre-planned literature search in MEDLINE Pubmed (1946 to present), EMBASE Ovid (1974 to present), and CINAHL (1981 to present) in July 2019 and updated in 2021. The key concepts used in the searches were "long-term care", "guidelines", "implementation", "barriers", and "facilitators". The key concepts were combined with the Boolean operator AND, and the search words within each concept were combined with OR. The full search strategy can be found in Additional file 2.

\section{Selection of studies}

All titles and abstracts were screened by two team members (CM and $\mathrm{YB}$ ) using a pilot-tested form and were included if they met our inclusion criteria as described above. We excluded articles that were not written in English, reported on implementation of guidelines that were not evidence-based (i.e., the article did not demonstrate that the guideline was developed through systematic review of literature), clinical commentaries, editorials, legal cases, letters, newspaper articles, abstracts, or unpublished literature. After title and abstract screening, the full texts of relevant articles were screened independently by the same two reviewers using a pilottested form. Disagreements were arbitrated by a third party.

\section{Data extraction}

Two team members ( $\mathrm{CM}$ and $\mathrm{YB}$ ) independently extracted and charted the following data in duplicate using a pilot-tested data extraction form: study description (title, author, country, province/state/region, design, 
objectives, data collection methods, data analysis methods, name of guidelines examined, health topic of guideline examined, behaviour change framework, model, or theory used), individual participant description (profession(s), number, mean age, sex, sampling technique, response rate), LTC home description (number, size, ownership, rurality), and results/findings (identified barriers and facilitators). Data for the study results were extracted verbatim from the text under the heading "results" or "findings" where authors identified barriers and facilitators (or a synonym, e.g., challenges or supports for change) to implementation of the guidelines examined.

\section{Assessing the methodological limitations of included studies}

The validity, robustness, and applicability of each included study was appraised by two team members (CM and $\mathrm{PH}$ ) independently and in duplicate using the Critical Appraisal Skills Programme (CASP) Checklist [18]. Consensus between the two reviewers was required, and any discrepancies were adjudicated by a third party. No studies were weighted or excluded based on the appraisal results.

\section{Data management, analysis, and synthesis}

Our synthesis follows the three-stage Thomas and Harden approach to inductive thematic synthesis [19]. We completed two steps of this process, as our primary aim was to produce descriptive themes of barriers and facilitators to guideline implementation across different health guidelines to then map on the COM-B components. After extracting the reported barriers and facilitators, two team members ( $\mathrm{CM}$ and $\mathrm{YB})$ created a codebook that was grouped into recurrent themes (e.g., resources, staffing issues). The two team members then independently and in duplicate coded each extracted barrier and facilitator with the themes from the code book. If new codes emerged, they were added iteratively to the code book and the barriers and facilitators were re-themed accordingly. The frequency of the themes was tallied as the number of times the theme was mentioned across the included articles. Finally, the themes were mapped onto the COM-B components of the Behaviour Change Wheel by the two team members independently and in duplicate. Based on a synthesis of 19 previously published behaviour change frameworks, the Behaviour Change Wheel provides tables that link the central COM-B components to potential knowledge translation intervention functions based on their expected effectiveness in relation to the barriers and facilitators. For example, if physical opportunity is a barrier, then training, restriction, environmental restructuring, and enablement are potential intervention functions. Potential knowledge translation intervention functions were listed with their associated barriers and facilitators and COM-B components. Any discrepancies between the two members were resolved by a third party. All data analysis and synthesis were performed in Microsoft Excel. Table 1 provide definitions for the COM-B components and knowledge translation intervention functions as outlined by the Behaviour Change Wheel.

\section{Assessing our confidence in the review findings}

Two review authors (CM and $\mathrm{PH})$ assessed the level of confidence for each finding using the GRADE-CERQual [20]. GRADE-CERQual assesses confidence in the evidence based on four key components: methodological limitations of included studies, coherence of the review findings, adequacy of the data contributing to a review finding, and relevance of the included studies to the review question. After assessing each of the four components, we made a judgement about the overall confidence in the evidence supporting the review finding and report it as high, moderate, low, or very low. The final assessment was based on consensus among the two review authors. All findings started as high confidence and were graded down if there were important concerns regarding any of the GRADE-CERQual components.

\section{Summary of qualitative findings table and evidence profile}

We present summaries of the findings and our assessments of confidence in these findings in the Summary of qualitative findings table (Table 3). We present detailed descriptions of our confidence assessment in an Evidence Profile (Additional file 3).

\section{Review author reflexivity}

The authors of this article are a multidisciplinary group of researchers and clinicians focused on geriatrics and improving care provision in LTC. They have engaged in several research studies in LTC including assessment of barriers and facilitators to implementation of practices, development of guidelines, knowledge translation, and randomized controlled trials. Since we have prior experience assessing barriers and facilitators in the LTC setting, some biases may exist as we may have preconceived ideas of what barriers and facilitators exist. Included studies that were conducted by one of the authors of the current paper were analyzed by two team members who were not authors of the included studies.

\section{Findings}

\section{Results of the search}

After screening 2680 articles, 33 that were published between 2004 and 2020 were included in the analyses (Fig. 1). 
Table 1 Definitions of the COM-B constructs and intervention functions as outlined by the Behaviour Change Wheel [7]

\begin{tabular}{|c|c|}
\hline & Definition \\
\hline \multicolumn{2}{|l|}{ COM-B construct } \\
\hline Physical capability & Physical skill, strength, or stamina \\
\hline $\begin{array}{l}\text { Psychological } \\
\text { capability }\end{array}$ & Knowledge or psychological skills, strength, or stamina to engage in the necessary mental processes \\
\hline Physical opportunity & Opportunity afforded by the environment involving time, resources, locations, cues, physical affordance \\
\hline Social opportunity & $\begin{array}{l}\text { Opportunity afforded by the interpersonal influences, social cues and cultural norms that influence the way that we think } \\
\text { about things }\end{array}$ \\
\hline Reflective motivation & Reflective processes involving plans (self-conscious intentions) and evaluations (beliefs about what is good and bad) \\
\hline $\begin{array}{l}\text { Automatic } \\
\text { motivation }\end{array}$ & $\begin{array}{l}\text { Automatic processes involving emotional reactions, desires (wants and needs), impulses, inhibitions, drive states, and } \\
\text { reflex responses }\end{array}$ \\
\hline \multicolumn{2}{|l|}{ Intervention function } \\
\hline $\begin{array}{l}\text { Environmental } \\
\text { restructuring }\end{array}$ & Changing the physical or social context \\
\hline Restrictions & $\begin{array}{l}\text { Using rules to reduce the opportunity to engage in the target behaviour (or to increase the target behaviour by } \\
\text { reducing the opportunity to engage in competing behaviours) }\end{array}$ \\
\hline Education & Increasing knowledge or understanding \\
\hline Persuasion & Using communication to induce positive or negative feelings to stimulate action \\
\hline Incentivisation & Creating an expectation of reward \\
\hline Coercion & Creating an expectation of punishment or cost \\
\hline Training & Imparting skills \\
\hline Enablement & $\begin{array}{l}\text { Increasing means/reducing barriers to increase capability (beyond education and training) or opportunity (beyond } \\
\text { environmental restructuring) }\end{array}$ \\
\hline Modeling & Provide an example for people to aspire to or imitate \\
\hline
\end{tabular}

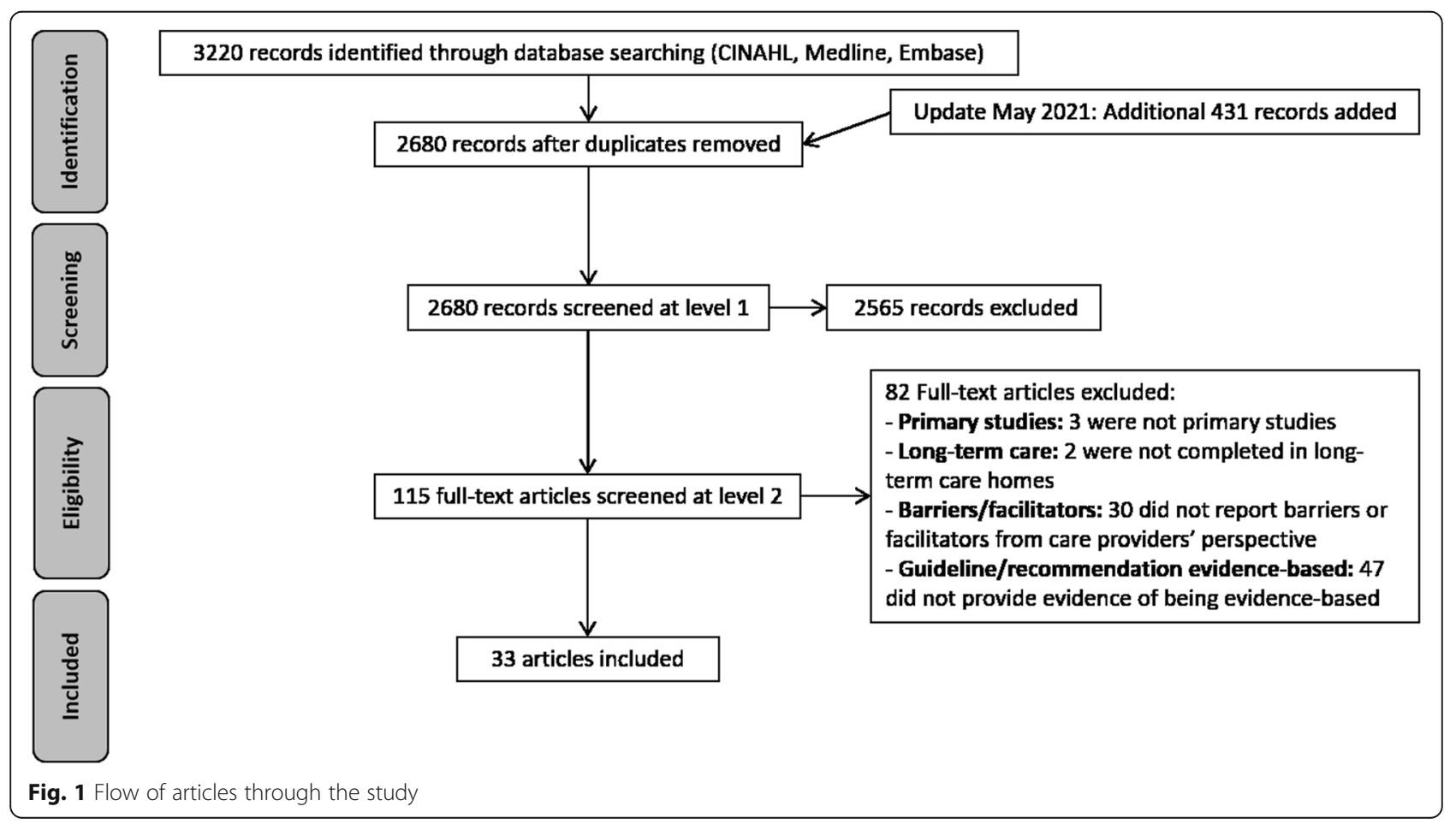




\section{Description of the studies}

Most studies were conducted in Canada and Australia, with much fewer in the Netherlands, the USA, England, Sweden, Germany, South Korea, and Belgium (Table 2). A wide range of guidelines were examined, with the most frequent being oral health, medication reviews, and pain protocols. A variety of study designs were employed including qualitative studies, mixed method, multiple case studies, and process evaluations. Focus groups, interviews, and document analysis were the most frequent data collection methods, and thematic or content analysis was used to analyze data for $73 \%$ of included studies. Only six studies used a behaviour change framework, model, or theory to guide their work which included the framework developed by Greenhalgh et al. (Capability, Opportunity, and Motivation), Organizational Readiness for Change, Theoretical Domains Framework, Organization Learning Theory, Promoting Action in on Research Implementation in Health Services, and Normalization Process Theory.

Included studies recruited 12 to 500 LTC home staff from a variety of professions including nursing, medicine, management, rehabilitation (e.g., physical and occupational therapy), pharmacy, and food services (Table 3). Many studies did not report the age or sex of their participants. For those that did, the mean age of included staff ranged from 38 to 54 years, and the percentage of participants who were female ranged from $46 \%$ to $100 \%$. Convenience and purposeful sampling were the most common methods of recruitment. At the LTC home level, the number of homes included ranged from 2 to 120 , and the number of residents per home ranged from 40 to 251; though many studies did not report these values $(11 \%$ did not report number of homes, $46 \%$ did not report number of residents per home). Similarly, more than half (58\%) of the included studies did not report the ownership or rurality of the included homes.

\section{Methodological limitations of the studies}

Most studies had a clear research aim which was appropriately addressed through a qualitative research design. Likewise, most studies employed appropriate recruitment strategies and data were collected in a way that addressed the research question. In some studies, the description of data analysis techniques was limited. Overall, we found poor reporting of research reflexivity across most of the included studies. Details of the assessments of methodological limitations for individual studies are found in Additional file 4.

\section{Confidence in the review findings}

We had moderate or high confidence in all but one of our review findings. Confidence was most often downgraded due to concerns with methodological limitations including a lack of discussion about credibility of qualitative findings and a lack of reflexivity. The data was almost always relevant as most studies examined our phenomena and population of interest. The full CERQual evidence profile can be found in Additional file 3.

\section{Review findings}

The line-by-line thematic analysis of barriers and facilitators is found in Additional file 5. Table 4 provides a summary of the identified barrier and facilitator themes, their definitions and frequency, the articles contributing to the theme, and the CERQual assessment and explanation of confidence in the findings. The most frequently identified barriers and facilitators were consistent across guideline topics, while others were more specific to the content of the guideline. For example, nearly all articles identified time constraints and inadequate staffing (high confidence), and cost and lack of resources (high confidence) as barriers. However, guideline impracticality (high confidence) and taking a reactive approach (moderate confidence) were only identified in articles that discussed physical activity, influenza immunization, pneumonia treatment, and heart failure. In some instances, barriers and facilitators were opposites of each other, with barriers being actual and facilitators being perceived. For example, if time and money were an identified barrier, the staff perceived they could more easily implement the guideline with more time and resources (facilitator). However, some facilitators were also actual. For example, champions to promote implementation of the guidelines within the home was an actual facilitator in several articles.

Physical and social opportunity were the COM-B components that the identified barriers and facilitators mapped onto most frequently (Table 5). Within physical and social opportunity, time constraints and inadequate staffing (high confidence), cost and lack of resources (high confidence), and lack of teamwork (high confidence) and organizational support (high confidence) were frequently reported barriers, while leadership and champions (high confidence), well designed strategies, protocols, and resources (high confidence), and adequate services, resources and time (high confidence) were frequent facilitators. Training, restriction, environmental restructuring, modelling, and enablement are knowledge translation intervention functions suggested by the Behaviour Change Wheel to overcome barriers associated with physical and social opportunity. The COM-B component of psychological capability represented knowledge gaps (high confidence) as a barrier and adequate knowledge and education (high confidence) as a facilitator. Education, training, environmental restructuring, modeling, and enablement are knowledge translation intervention functions suggested by the Behaviour 
Table 2 Characteristics of included studies

\begin{tabular}{|c|c|c|c|c|c|c|c|c|}
\hline Study & Year & $\begin{array}{l}\text { Country } \\
\text { (Province, } \\
\text { state, or } \\
\text { region) }\end{array}$ & Study design & Study objectives & $\begin{array}{l}\text { Data collection } \\
\text { methods }\end{array}$ & Analysis & $\begin{array}{l}\text { Guidelines and } \\
\text { health topic } \\
\text { examined }\end{array}$ & $\begin{array}{l}\text { Behaviour } \\
\text { change } \\
\text { framework, } \\
\text { model, or } \\
\text { theory }\end{array}$ \\
\hline $\begin{array}{l}\text { Phipps et al. } \\
\text { [36] }\end{array}$ & 2019 & $\begin{array}{l}\text { England } \\
\text { (South East) }\end{array}$ & $\begin{array}{l}\text { Qualitative } \\
\text { study }\end{array}$ & $\begin{array}{l}\text { To explore what } \\
\text { factors impact the } \\
\text { ability of clinicians } \\
\text { to manage care } \\
\text { home flu } \\
\text { outbreaks } \\
\text { according to } \\
\text { national guidelines } \\
\text { and highlight } \\
\text { opportunities for } \\
\text { change }\end{array}$ & $\begin{array}{l}\text { Collected notes } \\
\text { written (from } \\
\text { discussions) } \\
\text { during responses } \\
\text { to outbreaks, } \\
\text { presentations on } \\
\text { influenza at } \\
\text { stakeholder } \\
\text { engagement } \\
\text { events }\end{array}$ & $\begin{array}{l}\text { Identified and } \\
\text { matched } \\
\text { codes to } \\
\text { themes- } \\
\text { capability, } \\
\text { opportunity, } \\
\text { and } \\
\text { organizational } \\
\text { factors from } \\
\text { framework }\end{array}$ & $\begin{array}{l}\text { National } \\
\text { guidelines } \\
\text { supporting } \\
\text { antiviral use }\end{array}$ & $\begin{array}{l}\text { Framework } \\
\text { developed by } \\
\text { Greenhalgh } \\
\text { et al., capability, } \\
\text { opportunity, } \\
\text { and motivation }\end{array}$ \\
\hline $\begin{array}{l}\text { Abraham } \\
\text { et al. [37] }\end{array}$ & 2019 & $\begin{array}{l}\text { Germany } \\
\text { (Varied) }\end{array}$ & $\begin{array}{l}\text { Process } \\
\text { evaluation } \\
\text { subcomponent } \\
\text { of a pragmatic } \\
\text { cluster } \\
\text { randomized } \\
\text { controlled trial }\end{array}$ & $\begin{array}{l}\text { To systematically } \\
\text { document the } \\
\text { implementation } \\
\text { process and } \\
\text { describe barriers } \\
\text { and facilitators }\end{array}$ & $\begin{array}{l}\text { Structured } \\
\text { interviews and } \\
\text { focus groups }\end{array}$ & Not reported & $\begin{array}{l}\text { IMPRINT_-to } \\
\text { reduce physical } \\
\text { restraint use }\end{array}$ & None \\
\hline $\begin{array}{l}\text { Villarosa } \\
\text { et al. [38] }\end{array}$ & 2018 & $\begin{array}{l}\text { Australia } \\
\text { (New South } \\
\text { Wales) }\end{array}$ & $\begin{array}{l}\text { Exploratory } \\
\text { qualitative study }\end{array}$ & $\begin{array}{l}\text { To explore the } \\
\text { perceptions of } \\
\text { care staff towards } \\
\text { the provision of } \\
\text { oral health care } \\
\text { following } \\
\text { implementation of } \\
\text { a new care model: } \\
\text { (1) examine the } \\
\text { perceptions of the } \\
\text { care staff } \\
\text { regarding oral } \\
\text { health care } \\
\text { practices; ( } 2 \text { ) } \\
\text { ascertain the } \\
\text { needs and } \\
\text { recommendations } \\
\text { of care staff in } \\
\text { relation to } \\
\text { improving the } \\
\text { delivery of oral } \\
\text { health care. }\end{array}$ & Focus group & $\begin{array}{l}\text { Inductive } \\
\text { thematic } \\
\text { analysis }\end{array}$ & $\begin{array}{l}\text { Better oral health } \\
\text { in Residential } \\
\text { Aged Care } \\
\text { programme }\end{array}$ & None \\
\hline $\begin{array}{l}\text { Huhtinen } \\
\text { et al. [39] }\end{array}$ & 2018 & $\begin{array}{l}\text { Australia } \\
\text { (Sydney) }\end{array}$ & Mixed method & $\begin{array}{l}\text { To identify the } \\
\text { perceived barriers } \\
\text { to the } \\
\text { implementation of } \\
\text { the Australian } \\
\text { guidelines on } \\
\text { influenza outbreak } \\
\text { management with } \\
\text { staff in an inner- } \\
\text { city Sydney region }\end{array}$ & $\begin{array}{l}\text { Telephone } \\
\text { interview using a } \\
\text { semi-structured } \\
\text { questionnaire }\end{array}$ & $\begin{array}{l}\text { Thematic } \\
\text { analysis }\end{array}$ & $\begin{array}{l}\text { National } \\
\text { Guidelines for the } \\
\text { Prevention, } \\
\text { Control and Public } \\
\text { Health } \\
\text { Management of } \\
\text { Influenza } \\
\text { Outbreaks in } \\
\text { Residential Care } \\
\text { Facilities in } \\
\text { Australia }\end{array}$ & None \\
\hline $\begin{array}{l}\text { Nilsen et al. } \\
{[40]}\end{array}$ & 2018 & $\begin{array}{l}\text { Sweden } \\
\text { (Southern } \\
\text { region) }\end{array}$ & $\begin{array}{l}\text { Qualitative } \\
\text { study }\end{array}$ & $\begin{array}{l}\text { To identify barriers } \\
\text { and facilitators to } \\
\text { implementing } \\
\text { evidence-based } \\
\text { palliative care in } \\
\text { the nursing } \\
\text { homes }\end{array}$ & $\begin{array}{l}\text { Semi-structured } \\
\text { interviews }\end{array}$ & $\begin{array}{l}\text { Content } \\
\text { analysis. Codes } \\
\text { were } \\
\text { compared } \\
\text { with } \\
\text { categories } \\
\text { outlined in the } \\
\text { Organizational } \\
\text { Readiness for } \\
\text { Change } \\
\text { Framework }\end{array}$ & $\begin{array}{l}\text { World Health } \\
\text { Organization } \\
\text { guidelines on } \\
\text { palliative care }\end{array}$ & $\begin{array}{l}\text { Organizational } \\
\text { Readiness for } \\
\text { Change }\end{array}$ \\
\hline
\end{tabular}


Table 2 Characteristics of included studies (Continued)

\begin{tabular}{|c|c|c|c|c|c|c|c|c|}
\hline Study & Year & $\begin{array}{l}\text { Country } \\
\text { (Province, } \\
\text { state, or } \\
\text { region) }\end{array}$ & Study design & Study objectives & $\begin{array}{l}\text { Data collection } \\
\text { methods }\end{array}$ & Analysis & $\begin{array}{l}\text { Guidelines and } \\
\text { health topic } \\
\text { examined }\end{array}$ & $\begin{array}{l}\text { Behaviour } \\
\text { change } \\
\text { framework, } \\
\text { model, or } \\
\text { theory }\end{array}$ \\
\hline $\begin{array}{l}\text { DuBeau } \\
\text { et al. [41] }\end{array}$ & 2007 & USA (Kansas) & Mixed method & $\begin{array}{l}\text { To survey nursing } \\
\text { home staff and } \\
\text { state surveyors } \\
\text { regarding } \\
\text { attitudes about } \\
\text { perceived and/or } \\
\text { experienced } \\
\text { barriers and } \\
\text { challenges to } \\
\text { implementing } \\
\text { F315 compliance }\end{array}$ & $\begin{array}{l}\text { Questionnaire } \\
\text { survey with Likert } \\
\text { type responses } \\
\text { and open-ended } \\
\text { questions }\end{array}$ & $\begin{array}{l}\text { Inductive } \\
\text { manifest and } \\
\text { latent content } \\
\text { analysis based } \\
\text { on grounded } \\
\text { theory }\end{array}$ & $\begin{array}{l}\text { F315 tag: } \\
\text { guidance for } \\
\text { meeting } \\
\text { compliance in } \\
\text { managing and } \\
\text { evaluating urinary } \\
\text { incontinence and } \\
\text { urinary catheters }\end{array}$ & None \\
\hline $\begin{array}{l}\text { Birney et al. } \\
\text { [42] }\end{array}$ & 2016 & $\begin{array}{l}\text { Canada } \\
\text { (Alberta) }\end{array}$ & $\begin{array}{l}\text { Exploratory } \\
\text { qualitative study }\end{array}$ & $\begin{array}{l}\text { To understand } \\
\text { how four LTC } \\
\text { facilities in Alberta } \\
\text { have implemented } \\
\text { medication } \\
\text { reviews for the } \\
\text { Appropriate Use } \\
\text { of Antipsychotic } \\
\text { Initiative }\end{array}$ & $\begin{array}{l}\text { Semi-structured } \\
\text { interviews and } \\
\text { observations }\end{array}$ & $\begin{array}{l}\text { Thematic } \\
\text { analysis }\end{array}$ & $\begin{array}{l}\text { Alberta Guideline } \\
\text { on the } \\
\text { Appropriate Use } \\
\text { of Antipsychotic } \\
\text { Medications }\end{array}$ & None \\
\hline $\begin{array}{l}\text { Fallon et al. } \\
\text { [43] }\end{array}$ & 2006 & $\begin{array}{l}\text { Australia (City } \\
\text { of } \\
\text { Toowoomba) }\end{array}$ & $\begin{array}{l}\text { Quality } \\
\text { improvement } \\
\text { study }\end{array}$ & $\begin{array}{l}\text { To identify barriers } \\
\text { to implementation } \\
\text { of evidence-based } \\
\text { recommendations } \\
\text { and strategies to } \\
\text { overcome these } \\
\text { barriers }\end{array}$ & $\begin{array}{l}\text { Semi-structured } \\
\text { focus group }\end{array}$ & $\begin{array}{l}\text { Thematic } \\
\text { analysis }\end{array}$ & $\begin{array}{l}\text { Evidence-based } \\
\text { recommendations } \\
\text { for oral health }\end{array}$ & None \\
\hline $\begin{array}{l}\text { Baert et al. } \\
\text { [44] }\end{array}$ & 2016 & $\begin{array}{l}\text { Belgium } \\
\text { (Flanders) }\end{array}$ & Mixed method & $\begin{array}{l}\text { To identify barriers } \\
\text { as well as } \\
\text { motivators for } \\
\text { organizing } \\
\text { physical activity in } \\
\text { LTC homes } \\
\text { according to } \\
\text { administrators on } \\
\text { the different levels } \\
\text { of the } \\
\text { socioecological } \\
\text { model. A } \\
\text { secondary goal } \\
\text { was examining } \\
\text { their knowledge } \\
\text { of the guidelines } \\
\text { regarding physical } \\
\text { activity and to } \\
\text { reveal potential } \\
\text { motivators and } \\
\text { barriers for the } \\
\text { implementation of } \\
\text { these guidelines }\end{array}$ & $\begin{array}{l}\text { Questionnaire } \\
\text { and interviews }\end{array}$ & $\begin{array}{l}\text { Deductive } \\
\text { qualitative } \\
\text { content } \\
\text { analysis } \\
\text { (Interviews) }\end{array}$ & $\begin{array}{l}\text { World Health } \\
\text { Organization } \\
\text { guidelines for } \\
\text { physical activity in } \\
\text { older adults }\end{array}$ & None \\
\hline $\begin{array}{l}\text { Alamri et al. } \\
\text { [45] }\end{array}$ & 2015 & $\begin{array}{l}\text { Canada } \\
\text { (Ontario) }\end{array}$ & $\begin{array}{l}\text { Qualitative } \\
\text { study }\end{array}$ & $\begin{array}{l}\text { To identify } \\
\text { potential barriers } \\
\text { to evidence-based } \\
\text { practices for } \\
\text { osteoporosis and } \\
\text { fracture preven- } \\
\text { tion in LTC } \\
\text { settings }\end{array}$ & $\begin{array}{l}\text { Action plan } \\
\text { worksheet } \\
\text { completed by } \\
\text { LTC staff in the } \\
\text { control arm of an } \\
\text { intervention study }\end{array}$ & $\begin{array}{l}\text { Deductive and } \\
\text { inductive } \\
\text { thematic } \\
\text { analysis }\end{array}$ & $\begin{array}{l}\text { Clinical practice } \\
\text { guidelines for the } \\
\text { diagnosis and } \\
\text { management of } \\
\text { osteoporosis in } \\
\text { Canada }\end{array}$ & $\begin{array}{l}\text { Theoretical } \\
\text { Domains } \\
\text { Framework }\end{array}$ \\
\hline $\begin{array}{l}\text { Kaasalainen } \\
\text { et al. [46] }\end{array}$ & 2014 & $\begin{array}{l}\text { Canada } \\
\text { (Ontario) }\end{array}$ & $\begin{array}{l}\text { Exploratory, } \\
\text { multiple-case } \\
\text { study }\end{array}$ & $\begin{array}{l}\text { What barriers and } \\
\text { facilitators are } \\
\text { encountered by }\end{array}$ & $\begin{array}{l}\text { Diaries recording } \\
\text { strategies, barriers, } \\
\text { facilitators; }\end{array}$ & $\begin{array}{l}\text { Thematic } \\
\text { analysis }\end{array}$ & $\begin{array}{l}\text { Interdisciplinary } \\
\text { pain protocol by } \\
\text { Kaasalainen et al. }\end{array}$ & None \\
\hline
\end{tabular}


Table 2 Characteristics of included studies (Continued)

\begin{tabular}{|c|c|c|c|c|c|c|c|c|}
\hline Study & Year & $\begin{array}{l}\text { Country } \\
\text { (Province, } \\
\text { state, or } \\
\text { region) }\end{array}$ & Study design & Study objectives & $\begin{array}{l}\text { Data collection } \\
\text { methods }\end{array}$ & Analysis & $\begin{array}{l}\text { Guidelines and } \\
\text { health topic } \\
\text { examined }\end{array}$ & $\begin{array}{l}\text { Behaviour } \\
\text { change } \\
\text { framework, } \\
\text { model, or } \\
\text { theory }\end{array}$ \\
\hline & & & & $\begin{array}{l}\text { the clinical nurse } \\
\text { specialists and } \\
\text { nurse practitioners } \\
\text { in changing team } \\
\text { practice related to } \\
\text { implementing a } \\
\text { pain protocol? }\end{array}$ & $\begin{array}{l}\text { participant } \\
\text { observation and } \\
\text { field notes by } \\
\text { research assistant; } \\
\text { interviews and } \\
\text { focus groups }\end{array}$ & & 2012 & \\
\hline $\begin{array}{l}\text { Vikstrom } \\
\text { et al. [47] }\end{array}$ & 2015 & $\begin{array}{l}\text { Sweden } \\
\text { (Stockholm) }\end{array}$ & $\begin{array}{l}\text { Implementation } \\
\text { study }\end{array}$ & $\begin{array}{l}\text { To outline the } \\
\text { nursing home staff } \\
\text { experiences } \\
\text { during the first } \\
\text { year of } \\
\text { implementation of } \\
\text { guidelines for care } \\
\text { of people with } \\
\text { dementia }\end{array}$ & $\begin{array}{l}\text { Reflective } \\
\text { seminars- } \\
\text { detailed notes } \\
\text { with experiential } \\
\text { data relating to } \\
\text { participant } \\
\text { experiences in } 4 \\
\text { discussions and } \\
\text { written content/ } \\
\text { illustrations from } \\
\text { posters }\end{array}$ & $\begin{array}{l}\text { Inductive and } \\
\text { deductive } \\
\text { qualitative } \\
\text { content } \\
\text { analysis }\end{array}$ & $\begin{array}{l}\text { Sweden's national } \\
\text { evidence-based } \\
\text { guidelines for care } \\
\text { of people with } \\
\text { dementia }\end{array}$ & None \\
\hline $\begin{array}{l}\text { Strachan } \\
\text { et al. [48] }\end{array}$ & 2014 & $\begin{array}{l}\text { Canada } \\
\text { (Ontario) }\end{array}$ & $\begin{array}{l}\text { Descriptive } \\
\text { qualitative study } \\
\text { nested in phase } \\
2 \text { of a three- } \\
\text { phase mixed } \\
\text { methods } \\
\text { protocol }\end{array}$ & $\begin{array}{l}\text { To explore LTC } \\
\text { nurses' } \\
\text { experiences in } \\
\text { managing heart } \\
\text { failure }\end{array}$ & Focus group & $\begin{array}{l}\text { Manifest } \\
\text { content } \\
\text { analysis }\end{array}$ & $\begin{array}{l}\text { Canadian } \\
\text { Cardiovascular } \\
\text { Society Heart } \\
\text { Failure guidelines } \\
\text { in LTC }\end{array}$ & None \\
\hline $\begin{array}{l}\text { Lim et al. } \\
{[49]}\end{array}$ & 2014 & $\begin{array}{l}\text { Australia } \\
\text { (Victoria) }\end{array}$ & Not reported & $\begin{array}{l}\text { To explore the } \\
\text { attitudes and } \\
\text { perceptions of key } \\
\text { healthcare } \\
\text { providers towards } \\
\text { antimicrobial } \\
\text { stewardship } \\
\text { interventions in } \\
\text { Australian } \\
\text { residential aged } \\
\text { care facilities }\end{array}$ & $\begin{array}{l}\text { Interviews and } \\
\text { focus groups }\end{array}$ & $\begin{array}{l}\text { Thematic } \\
\text { analysis using } \\
\text { the framework } \\
\text { approach }\end{array}$ & $\begin{array}{l}\text { International } \\
\text { guidelines for } \\
\text { infection control } \\
\text { and prevention }\end{array}$ & None \\
\hline $\begin{array}{l}\text { Dellefield } \\
\text { et al. [50] }\end{array}$ & 2014 & $\begin{array}{l}\text { USA } \\
\text { (California) }\end{array}$ & $\begin{array}{l}\text { Exploratory } \\
\text { qualitative } \\
\text { interview }\end{array}$ & $\begin{array}{l}\text { To describe } \\
\text { nurses' } \\
\text { perceptions of } \\
\text { individual and } \\
\text { organization-level } \\
\text { factors influencing } \\
\text { performance of } \\
\text { pressure ulcer pre- } \\
\text { vention care in } 2 \\
\text { VHA Nursing } \\
\text { Home Community } \\
\text { Living Centers to } \\
\text { help identify exist- } \\
\text { ing factors per- } \\
\text { ceived as } \\
\text { facilitators and } \\
\text { barriers to deliver- } \\
\text { ing pressure ulcer } \\
\text { prevention care }\end{array}$ & $\begin{array}{l}\text { Semi-structured } \\
\text { interviews }\end{array}$ & $\begin{array}{l}\text { Content } \\
\text { analysis }\end{array}$ & $\begin{array}{l}\text { Evidence-based } \\
\text { guidelines for } \\
\text { prevention of } \\
\text { pressure ulcers }\end{array}$ & None \\
\hline $\begin{array}{l}\text { Berta et al. } \\
\text { [28] }\end{array}$ & 2013 & $\begin{array}{l}\text { Canada } \\
\text { (Ontario) }\end{array}$ & Survey & $\begin{array}{l}\text { To better } \\
\text { understand how } \\
\text { care protocols are } \\
\text { implemented in } \\
\text { LTC homes } \\
\text { operating in }\end{array}$ & $\begin{array}{l}\text { Pen and paper } \\
\text { survey }\end{array}$ & $\begin{array}{l}\text { Mean score of } \\
\text { importance }\end{array}$ & $\begin{array}{l}\text { Clinical practice } \\
\text { guidelines for } \\
\text { either preventative } \\
\text { skin care, wound/ } \\
\text { ulcer care, restraint } \\
\text { use, management }\end{array}$ & $\begin{array}{l}\text { Organizational } \\
\text { learning theory }\end{array}$ \\
\hline
\end{tabular}


Table 2 Characteristics of included studies (Continued)

\begin{tabular}{|c|c|c|c|c|c|c|c|c|}
\hline Study & Year & $\begin{array}{l}\text { Country } \\
\text { (Province, } \\
\text { state, or } \\
\text { region) }\end{array}$ & Study design & Study objectives & $\begin{array}{l}\text { Data collection } \\
\text { methods }\end{array}$ & Analysis & $\begin{array}{l}\text { Guidelines and } \\
\text { health topic } \\
\text { examined }\end{array}$ & $\begin{array}{l}\text { Behaviour } \\
\text { change } \\
\text { framework, } \\
\text { model, or } \\
\text { theory }\end{array}$ \\
\hline & & & & $\begin{array}{l}\text { Ontario, and to } \\
\text { learn what } \\
\text { processes, } \\
\text { structural } \\
\text { mechanisms, and } \\
\text { knowledge } \\
\text { sources are } \\
\text { relevant to their } \\
\text { implementation }\end{array}$ & & & $\begin{array}{l}\text { of incontinence, } \\
\text { management of } \\
\text { difficult } \\
\text { behaviours, and } \\
\text { antimicrobial } \\
\text { resistance }\end{array}$ & \\
\hline $\begin{array}{l}\text { Bamford } \\
\text { et al. [51] }\end{array}$ & 2012 & $\begin{array}{l}\text { England (Not } \\
\text { reported) }\end{array}$ & $\begin{array}{l}\text { Process } \\
\text { evaluation }\end{array}$ & $\begin{array}{l}\text { To explore } \\
\text { facilitators and } \\
\text { barriers to the use } \\
\text { of nutrition } \\
\text { guidelines in } \\
\text { residential care } \\
\text { homes }\end{array}$ & $\begin{array}{l}\text { Semi-structured } \\
\text { interviews, } \\
\text { informal } \\
\text { discussions, } \\
\text { nonparticipant } \\
\text { observation }\end{array}$ & $\begin{array}{l}\text { Thematic } \\
\text { analysis, } \\
\text { themes then } \\
\text { mapped onto } \\
\text { the } \\
\text { Normalization } \\
\text { Process } \\
\text { Framework }\end{array}$ & $\begin{array}{l}\text { UK Food } \\
\text { Standards Agency } \\
\text { nutrient and food- } \\
\text { based guidance } \\
\text { for older people in } \\
\text { residential care }\end{array}$ & $\begin{array}{l}\text { Normalization } \\
\text { Process Theory }\end{array}$ \\
\hline $\begin{array}{l}\text { Kaasalainen } \\
\text { et al. [52] }\end{array}$ & 2012 & $\begin{array}{l}\text { Canada } \\
\text { (Ontario) }\end{array}$ & Mixed method & $\begin{array}{l}\text { To evaluate } \\
\text { dissemination } \\
\text { strategies in } \\
\text { improving clinical } \\
\text { practice } \\
\text { behaviours (e.g., } \\
\text { documentation of } \\
\text { pain assessments, } \\
\text { use of pain } \\
\text { medications and } \\
\text { non- } \\
\text { pharmacological } \\
\text { interventions) } \\
\text { among health care } \\
\text { team members, } \\
\text { and the effective- } \\
\text { ness of the pain } \\
\text { protocol in redu- } \\
\text { cing pain in LTC } \\
\text { residents }\end{array}$ & $\begin{array}{l}\text { Focus group and } \\
\text { interviews }\end{array}$ & $\begin{array}{l}\text { Thematic } \\
\text { content } \\
\text { analysis }\end{array}$ & $\begin{array}{l}\text { The American } \\
\text { Medical Directors' } \\
\text { Association and } \\
\text { American } \\
\text { Geriatrics Society } \\
\text { best practice } \\
\text { guidelines for pain }\end{array}$ & None \\
\hline $\begin{array}{l}\text { Verkaik et al. } \\
\text { [53] }\end{array}$ & 2011 & $\begin{array}{l}\text { Netherlands } \\
\text { (Not } \\
\text { reported) }\end{array}$ & $\begin{array}{l}\text { Multiple case } \\
\text { study }\end{array}$ & $\begin{array}{l}\text { Which factors } \\
\text { facilitate or inhibit } \\
\text { successful } \\
\text { introduction of } \\
\text { the guideline in } \\
\text { psychogeriatric } \\
\text { nursing home } \\
\text { wards? Which } \\
\text { factors facilitate or } \\
\text { inhibit the } \\
\text { successful } \\
\text { application of the } \\
\text { guideline by CNAs } \\
\text { in their support of } \\
\text { residents with } \\
\text { comorbid } \\
\text { depression? }\end{array}$ & $\begin{array}{l}\text { Semi-structured } \\
\text { interviews, } \\
\text { memos, } \\
\text { evaluation forms, } \\
\text { activity plan } \\
\text { forms, training } \\
\text { reports } \\
\text { observations }\end{array}$ & $\begin{array}{l}\text { Qualitative } \\
\text { data analysis }\end{array}$ & $\begin{array}{l}\text { Depression in } \\
\text { Dementia }\end{array}$ & None \\
\hline $\begin{array}{l}\text { Berta et al. } \\
{[54]}\end{array}$ & 2010 & $\begin{array}{l}\text { Canada } \\
\text { (Ontario) }\end{array}$ & $\begin{array}{l}\text { Multiple case } \\
\text { study }\end{array}$ & $\begin{array}{l}\text { To explore the } \\
\text { translational } \\
\text { process that } \\
\text { emerges within } \\
\text { Ontario long-term } \\
\text { care homes with } \\
\text { the adoption and } \\
\text { implementation of }\end{array}$ & $\begin{array}{l}\text { Semi-structured } \\
\text { interviews, focus } \\
\text { groups }\end{array}$ & $\begin{array}{l}\text { Template } \\
\text { analysis via } \\
\text { constant } \\
\text { comparative } \\
\text { analysis }\end{array}$ & $\begin{array}{l}\text { Clinical practice } \\
\text { guidelines for } \\
\text { either preventative } \\
\text { skin care, wound/ } \\
\text { ulcer care, restraint } \\
\text { use, management } \\
\text { of incontinence, } \\
\text { management of }\end{array}$ & $\begin{array}{l}\text { Organizational } \\
\text { learning theory }\end{array}$ \\
\hline
\end{tabular}


Table 2 Characteristics of included studies (Continued)

\begin{tabular}{|c|c|c|c|c|c|c|c|c|}
\hline Study & Year & $\begin{array}{l}\text { Country } \\
\text { (Province, } \\
\text { state, or } \\
\text { region) }\end{array}$ & Study design & Study objectives & $\begin{array}{l}\text { Data collection } \\
\text { methods }\end{array}$ & Analysis & $\begin{array}{l}\text { Guidelines and } \\
\text { health topic } \\
\text { examined }\end{array}$ & $\begin{array}{l}\text { Behaviour } \\
\text { change } \\
\text { framework, } \\
\text { model, or } \\
\text { theory }\end{array}$ \\
\hline & & & & $\begin{array}{l}\text { evidence-based } \\
\text { clinical practice } \\
\text { guidelines }\end{array}$ & & & $\begin{array}{l}\text { difficult } \\
\text { behaviours, and } \\
\text { antimicrobial } \\
\text { resistance }\end{array}$ & \\
\hline $\begin{array}{l}\text { McConigley } \\
\text { et al. [55] }\end{array}$ & 2008 & $\begin{array}{l}\text { Australia } \\
\text { (Perth) }\end{array}$ & $\begin{array}{l}\text { Qualitative } \\
\text { study }\end{array}$ & $\begin{array}{l}\text { Identify barriers } \\
\text { and facilitators to } \\
\text { guideline } \\
\text { implementation } \\
\text { and strengths that } \\
\text { could assist in the } \\
\text { implementation } \\
\text { process }\end{array}$ & $\begin{array}{l}\text { Focus groups and } \\
\text { interviews }\end{array}$ & $\begin{array}{l}\text { Thematic } \\
\text { analysis }\end{array}$ & $\begin{array}{l}\text { Australian Pain } \\
\text { Society for } \\
\text { residents in } \\
\text { residential aged } \\
\text { care facilities }\end{array}$ & None \\
\hline $\begin{array}{l}\text { Cheek et al. } \\
\text { [56] }\end{array}$ & 2004 & $\begin{array}{l}\text { Australia } \\
\text { (South) }\end{array}$ & $\begin{array}{l}\text { Descriptive/ } \\
\text { exploratory } \\
\text { multimethod } \\
\text { multilayered } \\
\text { design }\end{array}$ & $\begin{array}{l}\text { To investigate the } \\
\text { factors that } \\
\text { influence the } \\
\text { implementation of } \\
\text { best practice } \\
\text { guidelines with } \\
\text { respect to quality } \\
\text { use of medicines } \\
\text { in residential aged } \\
\text { care facilities }\end{array}$ & $\begin{array}{l}\text { Critical Incident } \\
\text { Technique, focus } \\
\text { groups, and } \\
\text { nominal groups }\end{array}$ & Not reported & $\begin{array}{l}\text { Nursing Guidelines } \\
\text { for Medication } \\
\text { Management in } \\
\text { Nursing Homes } \\
\text { and Hostels, } \\
\text { Guidelines for } \\
\text { Medical Care of } \\
\text { Older Persons in } \\
\text { Nursing Homes } \\
\text { and Hostels, Best } \\
\text { Practice Model for } \\
\text { the Supply of } \\
\text { Pharmacy Services } \\
\text { to Residential Care } \\
\text { Facilities }\end{array}$ & None \\
\hline $\begin{array}{l}\text { Hilton et al. } \\
\text { [57] }\end{array}$ & 2016 & $\begin{array}{l}\text { Australia (not } \\
\text { reported) }\end{array}$ & Mixed method & $\begin{array}{l}\text { To determine the } \\
\text { views and } \\
\text { experiences of } \\
\text { nurses and care } \\
\text { staff in residential } \\
\text { care settings in } \\
\text { relation to (a) } \\
\text { implementing } \\
\text { best practice oral } \\
\text { care guidelines } \\
\text { with residents of } \\
\text { long-term care } \\
\text { setting who have } \\
\text { chronic disabling } \\
\text { health conditions } \\
\text { and (b) the bar- } \\
\text { riers and facilita- } \\
\text { tors to the } \\
\text { implementation of } \\
\text { common oral care } \\
\text { practices included } \\
\text { in clinical } \\
\text { guidelines }\end{array}$ & $\begin{array}{l}\text { Online survey and } \\
\text { focus group }\end{array}$ & $\begin{array}{l}\text { Thematic } \\
\text { content } \\
\text { analysis }\end{array}$ & $\begin{array}{l}\text { Several oral care } \\
\text { guidelines }\end{array}$ & None \\
\hline $\begin{array}{l}\text { Lau et al. } \\
\text { [58] }\end{array}$ & 2007 & $\begin{array}{l}\text { USA } \\
\text { (Michigan) }\end{array}$ & Not reported & $\begin{array}{l}\text { To examine the } \\
\text { importance of } \\
\text { work-related fac- } \\
\text { tors such as inter- } \\
\text { professional com- } \\
\text { munication, par- } \\
\text { ticipation in } \\
\text { decision making, } \\
\text { and relationships } \\
\text { among clinical } \\
\text { staff members, for } \\
\text { the adoption of }\end{array}$ & $\begin{array}{l}\text { Semi-structured } \\
\text { interviews }\end{array}$ & $\begin{array}{l}\text { Thematic } \\
\text { analysis }\end{array}$ & $\begin{array}{l}\text { Federal guidelines } \\
\text { on medication } \\
\text { delivery CMS- } \\
\text { mandated drug } \\
\text { regimen review } \\
\text { quality indicators, } \\
\text { modified Beers cri- } \\
\text { teria, and other } \\
\text { practice guide- } \\
\text { lines, such as } \\
\text { those issued by } \\
\text { the American }\end{array}$ & None \\
\hline
\end{tabular}


Table 2 Characteristics of included studies (Continued)

\begin{tabular}{|c|c|c|c|c|c|c|c|c|}
\hline Study & Year & $\begin{array}{l}\text { Country } \\
\text { (Province, } \\
\text { state, or } \\
\text { region) }\end{array}$ & Study design & Study objectives & $\begin{array}{l}\text { Data collection } \\
\text { methods }\end{array}$ & Analysis & $\begin{array}{l}\text { Guidelines and } \\
\text { health topic } \\
\text { examined }\end{array}$ & $\begin{array}{l}\text { Behaviour } \\
\text { change } \\
\text { framework, } \\
\text { model, or } \\
\text { theory }\end{array}$ \\
\hline & & & & $\begin{array}{l}\text { guidelines in nurs- } \\
\text { ing homes }\end{array}$ & & & $\begin{array}{l}\text { Medical Directors } \\
\text { Association }\end{array}$ & \\
\hline $\begin{array}{l}\text { Buss et al. } \\
{[59]}\end{array}$ & 2004 & $\begin{array}{l}\text { Netherlands } \\
\text { (Limburg, } \\
\text { Noord- } \\
\text { Brabant) }\end{array}$ & $\begin{array}{l}\text { Qualitative } \\
\text { study }\end{array}$ & $\begin{array}{l}\text { To elucidate the } \\
\text { views and beliefs } \\
\text { of health care } \\
\text { workers (especially } \\
\text { enrolled nurses) in } \\
\text { Dutch nursing } \\
\text { homes about } \\
\text { pressure ulcer } \\
\text { prevention and } \\
\text { about issues } \\
\text { related with } \\
\text { pressure ulcer } \\
\text { prevention }\end{array}$ & $\begin{array}{l}\text { Interviews, written } \\
\text { pressure } \\
\text { prevention } \\
\text { protocols }\end{array}$ & $\begin{array}{l}\text { Thematic } \\
\text { analysis }\end{array}$ & $\begin{array}{l}\text { Dutch National } \\
\text { Guidelines for } \\
\text { Pressure Ulcer } \\
\text { Prevention }\end{array}$ & None \\
\hline $\begin{array}{l}\text { Van der } \\
\text { Maaden } \\
\text { et al. [60] }\end{array}$ & 2017 & $\begin{array}{l}\text { Netherlands } \\
\text { (Not } \\
\text { reported) }\end{array}$ & $\begin{array}{l}\text { Process } \\
\text { evaluation }\end{array}$ & $\begin{array}{l}\text { To provide further } \\
\text { understanding on } \\
\text { the lack on an } \\
\text { intervention effect } \\
\text { in the cluster } \\
\text { randomized trial. }\end{array}$ & $\begin{array}{l}\text { Observation, } \\
\text { interviews, survey }\end{array}$ & $\begin{array}{l}\text { Content } \\
\text { analysis }\end{array}$ & $\begin{array}{l}\text { Practice guidelines } \\
\text { for optimal } \\
\text { symptom relief of } \\
\text { pneumonia for } \\
\text { residents with } \\
\text { dementia }\end{array}$ & None \\
\hline $\begin{array}{l}\text { Kong et al. } \\
{[61]}\end{array}$ & 2021 & $\begin{array}{l}\text { South Korea } \\
\text { (Seoul } \\
\text { Special City, } \\
\text { Gtyeonggi- } \\
\text { do, Incheon } \\
\text { Metropolitan } \\
\text { City, } \\
\text { Gangwon- } \\
\text { do) }\end{array}$ & $\begin{array}{l}\text { Qualitative } \\
\text { descriptive } \\
\text { study }\end{array}$ & $\begin{array}{l}\text { To describe } \\
\text { nursing home } \\
\text { staff's perceptions } \\
\text { of the barriers and } \\
\text { needs in } \\
\text { implementing } \\
\text { care for people } \\
\text { with dementia in } \\
\text { Korean nursing } \\
\text { homes }\end{array}$ & $\begin{array}{l}\text { Semi-structured } \\
\text { interviews }\end{array}$ & $\begin{array}{l}\text { Qualitative } \\
\text { content } \\
\text { analysis }\end{array}$ & $\begin{array}{l}\text { Person-centred } \\
\text { dementia care }\end{array}$ & None \\
\hline $\begin{array}{l}\text { Jeong et al. } \\
\text { [62] }\end{array}$ & 2020 & $\begin{array}{l}\text { South Korea } \\
\text { (Not } \\
\text { reported) }\end{array}$ & $\begin{array}{l}\text { Mixed methods } \\
\text { study }\end{array}$ & $\begin{array}{l}\text { To identify the } \\
\text { barriers to } \\
\text { implementation of } \\
\text { a CPG perceived } \\
\text { by healthcare } \\
\text { professionals }\end{array}$ & $\begin{array}{l}\text { Semi-structured } \\
\text { interviews }\end{array}$ & $\begin{array}{l}\text { Thematic } \\
\text { analysis }\end{array}$ & $\begin{array}{l}\text { Clinical practice } \\
\text { guidelines for } \\
\text { management of } \\
\text { delirium }\end{array}$ & None \\
\hline $\begin{array}{l}\text { Eldh et al. } \\
\text { [63] }\end{array}$ & 2020 & $\begin{array}{l}\text { England, } \\
\text { Ireland, } \\
\text { Netherlands, } \\
\text { Sweden (Not } \\
\text { reported) }\end{array}$ & $\begin{array}{l}\text { Cluster } \\
\text { randomized } \\
\text { controlled trial } \\
\text { with embedded } \\
\text { realist } \\
\text { evaluation }\end{array}$ & $\begin{array}{l}\text { To demonstrate } \\
\text { the added and } \\
\text { unique } \\
\text { contribution } \\
\text { observations } \\
\text { made in } \\
\text { comparison with } \\
\text { survey and } \\
\text { stakeholder } \\
\text { interviews in a } \\
\text { mixed method } \\
\text { implementation } \\
\text { study }\end{array}$ & $\begin{array}{l}\text { Non-participant } \\
\text { observations, } \\
\text { survey, and } \\
\text { interviews }\end{array}$ & $\begin{array}{l}\text { Content } \\
\text { analysis }\end{array}$ & $\begin{array}{l}\text { Continence } \\
\text { Management } \\
\text { Guidelines }\end{array}$ & $\begin{array}{l}\text { Promoting } \\
\text { Action on } \\
\text { Research } \\
\text { Implementation } \\
\text { in Health } \\
\text { Services } \\
\text { Framework }\end{array}$ \\
\hline $\begin{array}{l}\text { Cossette } \\
\text { et al. [64] }\end{array}$ & 2020 & $\begin{array}{l}\text { Canada } \\
\text { (Quebec) }\end{array}$ & $\begin{array}{l}\text { Prospective } \\
\text { closed cohort } \\
\text { supplemented } \\
\text { by a } \\
\text { development } \\
\text { evaluation }\end{array}$ & $\begin{array}{l}\text { To identify barriers } \\
\text { and enablers in } \\
\text { relation to the } \\
\text { long-term integra- } \\
\text { tion of the OPUS- } \\
\text { AP strategy in rou- } \\
\text { tine care }\end{array}$ & $\begin{array}{l}\text { Semi-structured } \\
\text { interviews }\end{array}$ & $\begin{array}{l}\text { Semi-inductive } \\
\text { thematic } \\
\text { analysis }\end{array}$ & $\begin{array}{l}\text { Appropriate use of } \\
\text { anti-psychotics for } \\
\text { behavioural and } \\
\text { psychological } \\
\text { symptoms of } \\
\text { dementia }\end{array}$ & None \\
\hline $\begin{array}{l}\text { Surr et al. } \\
{[65]}\end{array}$ & 2020 & $\begin{array}{l}\text { England } \\
\text { (West } \\
\text { Yorkshire, }\end{array}$ & $\begin{array}{l}\text { Pragmatic } \\
\text { cluster } \\
\text { randomized }\end{array}$ & $\begin{array}{l}\text { To examine the } \\
\text { perceived barriers } \\
\text { to and facilitators }\end{array}$ & $\begin{array}{l}\text { Semi-structured } \\
\text { interviews }\end{array}$ & $\begin{array}{l}\text { Framework } \\
\text { analysis }\end{array}$ & $\begin{array}{l}\text { Dementia Care } \\
\text { Mapping }\end{array}$ & None \\
\hline
\end{tabular}


Table 2 Characteristics of included studies (Continued)

\begin{tabular}{|c|c|c|c|c|c|c|c|c|}
\hline Study & Year & $\begin{array}{l}\text { Country } \\
\text { (Province, } \\
\text { state, or } \\
\text { region) }\end{array}$ & Study design & Study objectives & $\begin{array}{l}\text { Data collection } \\
\text { methods }\end{array}$ & Analysis & $\begin{array}{l}\text { Guidelines and } \\
\text { health topic } \\
\text { examined }\end{array}$ & $\begin{array}{l}\text { Behaviour } \\
\text { change } \\
\text { framework, } \\
\text { model, or } \\
\text { theory }\end{array}$ \\
\hline & & $\begin{array}{l}\text { Oxfordshire, } \\
\text { South } \\
\text { London) }\end{array}$ & $\begin{array}{l}\text { controlled trial } \\
\text { with a process } \\
\text { evaluation }\end{array}$ & $\begin{array}{l}\text { of intervention } \\
\text { implementation, } \\
\text { the mechanisms } \\
\text { of impact and the } \\
\text { perceived impacts } \\
\text { from the } \\
\text { perspective of } \\
\text { mappers, expert } \\
\text { mappers, } \\
\text { managers, staff, } \\
\text { residents and } \\
\text { relatives }\end{array}$ & & & & \\
\hline $\begin{array}{l}\text { Desveaux } \\
\text { et al. [66] }\end{array}$ & 2019 & $\begin{array}{l}\text { Canada } \\
\text { (Ontario) }\end{array}$ & $\begin{array}{l}\text { Qualitative } \\
\text { process } \\
\text { evaluation }\end{array}$ & $\begin{array}{l}\text { To examine } \\
\text { whether, how, and } \\
\text { why an academic } \\
\text { detailing } \\
\text { intervention could } \\
\text { improve evidence } \\
\text { uptake and (2) } \\
\text { identify perceived } \\
\text { changes that } \\
\text { occurred to inform } \\
\text { outcomes } \\
\text { appropriate for } \\
\text { quantitative } \\
\text { evaluation. }\end{array}$ & $\begin{array}{l}\text { Semi-structured } \\
\text { interviews }\end{array}$ & $\begin{array}{l}\text { Inductive } \\
\text { approach } \\
\text { within the } \\
\text { framework } \\
\text { method }\end{array}$ & $\begin{array}{l}\text { Fall prevention } \\
\text { guideline }\end{array}$ & None \\
\hline Walker [67] & 2019 & $\begin{array}{l}\text { Australia (Not } \\
\text { reported) }\end{array}$ & $\begin{array}{l}\text { Process } \\
\text { evaluation }\end{array}$ & $\begin{array}{l}\text { To report on } \\
\text { process outcomes } \\
\text { of the ViDAus } \\
\text { study evaluating } \\
\text { the feasibility of } \\
\text { this multifaceted, } \\
\text { interdisciplinary } \\
\text { knowledge } \\
\text { translation } \\
\text { intervention for } \\
\text { the } \\
\text { implementation of } \\
\text { vitamin D } \\
\text { supplement use in } \\
\text { residential aged } \\
\text { care facilities }\end{array}$ & Unclear & Not reported & $\begin{array}{l}\text { Vitamin D } \\
\text { supplementation } \\
\text { guidelines }\end{array}$ & $\begin{array}{l}\text { Promoting } \\
\text { Action on } \\
\text { Research } \\
\text { Implementation } \\
\text { in Health } \\
\text { Services } \\
\text { Framework }\end{array}$ \\
\hline
\end{tabular}

LTC long-term care

Change Wheel to overcome barriers associated with psychological capability. Finally, reflective and automatic motivation had barriers relating to conflict with clinical autonomy (high confidence), beliefs against the guideline (high confidence), moral distress (moderate confidence), reluctance to change (high confidence), emotional responses to work and confidence in skills (moderate confidence), and change fatigue (moderate confidence). Facilitators with respect to reflective and automatic motivation were having noticeable outcomes occur from guidelines implementation (moderate confidence), a sense of conviction that the guidelines are evidencebased and will demonstrate improvement (low confidence), and a positive emotional response to work and the intervention (high confidence). The Behaviour Change Wheel suggests training, education, persuasion, modelling, enablement, incentivization, coercion, and environmental restructuring as potential knowledge translation interventions to overcome automatic and reflective motivation.

\section{Review author reflexivity}

We previously described our initial positioning earlier (see review author reflexivity above). Throughout the review, our positioning remained the same. During analysis and writing of the discussion, we felt our findings confirmed our initial ideas about the most frequent barriers and facilitators. 


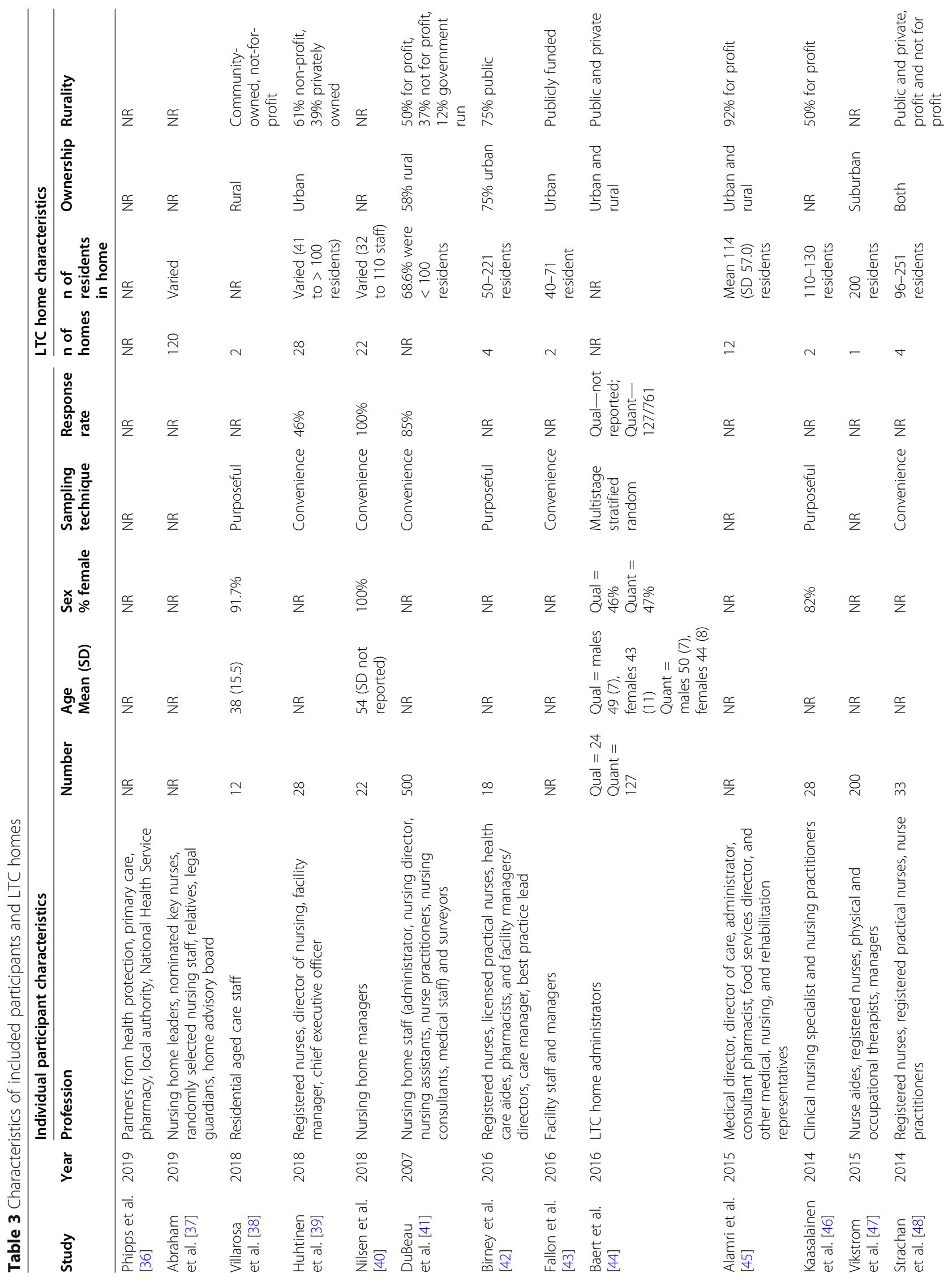




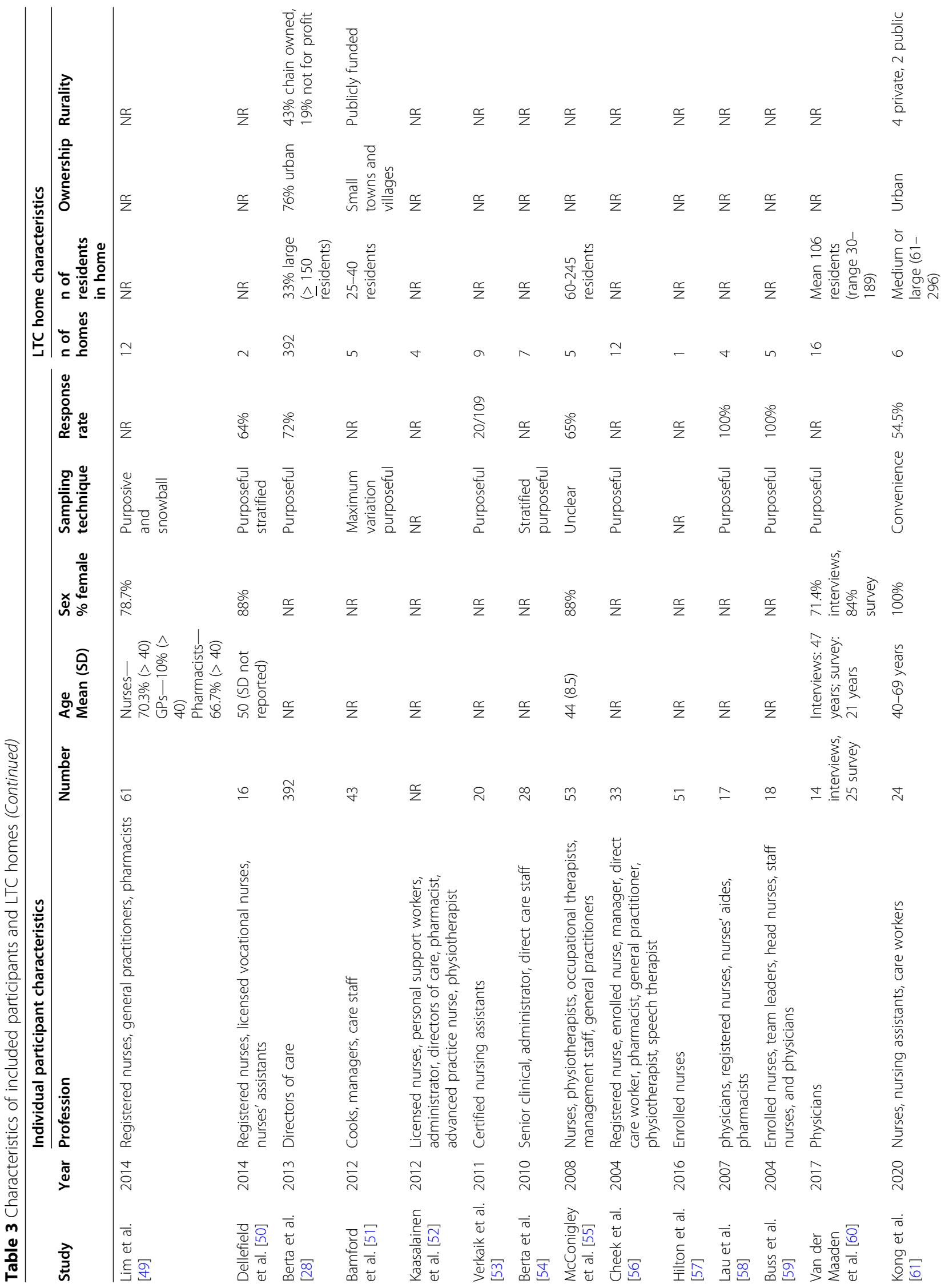




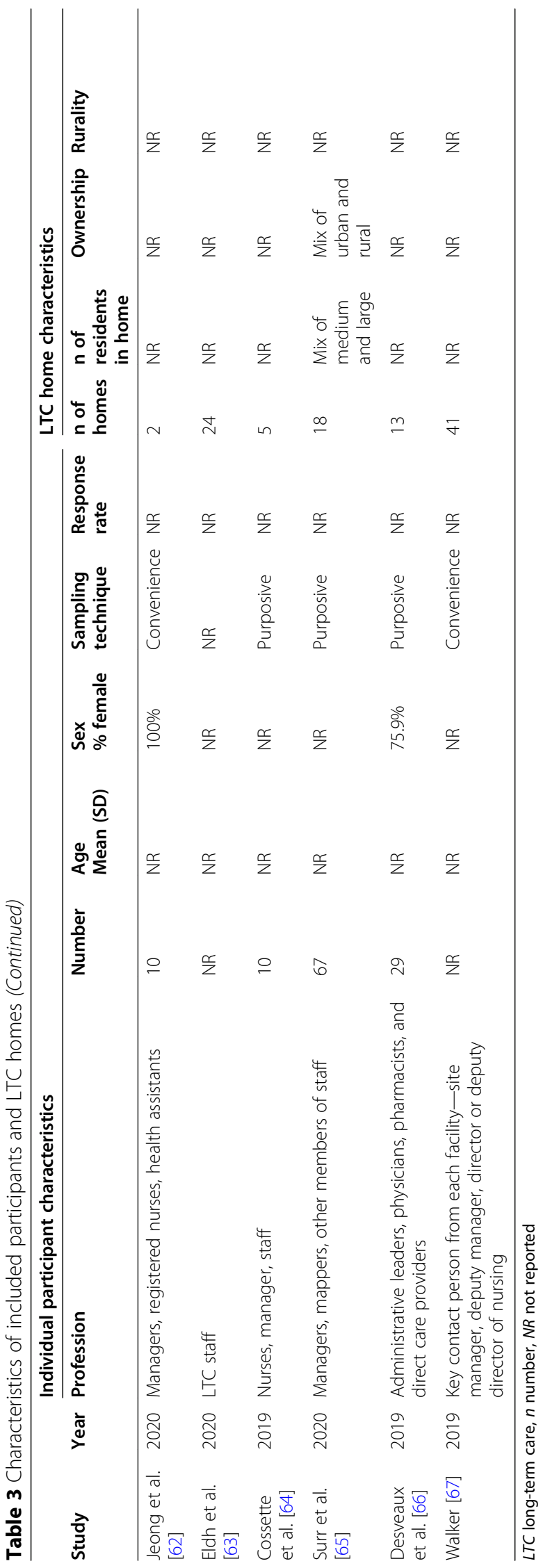


Table 4 GRADE-CERQual summary of qualitative review findings table: barriers and facilitators of implementing evidence-based guidelines in long-term care

\begin{tabular}{|c|c|c|c|c|c|}
\hline & Summary of review finding & $\begin{array}{l}\text { Contributing } \\
\text { articles }\end{array}$ & Frequency & $\begin{array}{l}\text { CERQual } \\
\text { Assessment of } \\
\text { confidence in } \\
\text { the evidence }\end{array}$ & Explanation of CERQual assessment \\
\hline \multirow[t]{14}{*}{ Barriers } & $\begin{array}{l}\text { Time constraints and inadequate staffing: } \\
\text { lack of time or personnel to carry out tasks } \\
\text { as indicated by the guideline }\end{array}$ & $\begin{array}{l}{[36,37,39-44} \\
47,49,53,55- \\
57,60,61,63- \\
65]\end{array}$ & 32 & $\begin{array}{l}\text { High } \\
\text { confidence }\end{array}$ & $\begin{array}{l}\text { Minor concerns regarding methodological } \\
\text { limitations, no or very minor concerns } \\
\text { regarding coherence, adequacy, and } \\
\text { relevance }\end{array}$ \\
\hline & $\begin{array}{l}\text { Knowledge gaps: inadequate training, } \\
\text { expertise, or awareness of the targeted } \\
\text { condition or guideline recommendations }\end{array}$ & $\begin{array}{l}{[36-41,43,48} \\
51,53,55-57 \\
61,62,65,67]\end{array}$ & 26 & $\begin{array}{l}\text { High } \\
\text { confidence }\end{array}$ & $\begin{array}{l}\text { Minor concerns regarding methodological } \\
\text { limitations, no or very minor concerns } \\
\text { regarding coherence, adequacy, and } \\
\text { relevance }\end{array}$ \\
\hline & $\begin{array}{l}\text { Cost and lack of resources: inadequate } \\
\text { financial and other resources (e.g., } \\
\text { equipment) to carry out tasks as indicated } \\
\text { by the guideline }\end{array}$ & $\begin{array}{l}{[36-42,44,45} \\
48,51,56,57 \\
62,63,65]\end{array}$ & 25 & $\begin{array}{l}\text { High } \\
\text { confidence }\end{array}$ & $\begin{array}{l}\text { Minor concerns regarding methodological } \\
\text { limitations, no or very minor concerns } \\
\text { regarding coherence, adequacy, and } \\
\text { relevance }\end{array}$ \\
\hline & $\begin{array}{l}\text { Lack of teamwork: lack of cooperation and } \\
\text { role coordination among the resident's } \\
\text { circle of care, including the LTC staff, family } \\
\text { members, clinicians, and specialized health } \\
\text { professionals }\end{array}$ & $\begin{array}{l}{[36,41,42,49} \\
51,53,55-59 \\
61,63,66,67]\end{array}$ & 22 & $\begin{array}{l}\text { High } \\
\text { confidence }\end{array}$ & $\begin{array}{l}\text { Minor concerns regarding methodological } \\
\text { limitations, no or very minor concerns } \\
\text { regarding coherence, adequacy, and } \\
\text { relevance }\end{array}$ \\
\hline & $\begin{array}{l}\text { Lack of organizational support: lack of } \\
\text { impetus for guideline implementation from } \\
\text { LTC home management. }\end{array}$ & $\begin{array}{l}{[37,38,43,44} \\
51,53,54,56 \\
57,60,64,65 \\
67]\end{array}$ & 20 & $\begin{array}{l}\text { High } \\
\text { confidence }\end{array}$ & $\begin{array}{l}\text { Minor concerns regarding methodological } \\
\text { limitations, no or very minor concerns } \\
\text { regarding coherence, adequacy, and } \\
\text { relevance }\end{array}$ \\
\hline & $\begin{array}{l}\text { Resident complexity: complex comorbidities } \\
\text { of LTC residents }\end{array}$ & $\begin{array}{l}{[36,37,44,50} \\
52,53,56,57 \\
63,67]\end{array}$ & 19 & $\begin{array}{l}\text { High } \\
\text { confidence }\end{array}$ & $\begin{array}{l}\text { Minor concerns regarding methodological } \\
\text { limitations, no or very minor concerns } \\
\text { regarding coherence, adequacy, and } \\
\text { relevance }\end{array}$ \\
\hline & $\begin{array}{l}\text { Compromised communication and } \\
\text { information flow: inadequate } \\
\text { communication of relevant information } \\
\text { between the resident, their family, staff, } \\
\text { and/or allied health professions }\end{array}$ & $\begin{array}{l}{[45,48,49,51} \\
52,54,56,58 \\
61,66]\end{array}$ & 15 & $\begin{array}{l}\text { High } \\
\text { confidence }\end{array}$ & $\begin{array}{l}\text { Minor concerns regarding methodological } \\
\text { limitations, no or very minor concerns } \\
\text { regarding coherence, adequacy, and } \\
\text { relevance }\end{array}$ \\
\hline & Staff turnover: frequent change in staff & $\begin{array}{l}{[37,41,43,47} \\
52,53,56,63 \\
65,67]\end{array}$ & 15 & $\begin{array}{l}\text { High } \\
\text { confidence }\end{array}$ & $\begin{array}{l}\text { Minor concerns regarding methodological } \\
\text { limitations, no or very minor concerns } \\
\text { regarding coherence, adequacy, and } \\
\text { relevance }\end{array}$ \\
\hline & $\begin{array}{l}\text { Belief against the guideline: distrust of the } \\
\text { guideline's recommendations and/or of its } \\
\text { evidence base }\end{array}$ & $\begin{array}{l}{[36,37,39,44} \\
51,52,58-60 \\
67]\end{array}$ & 15 & $\begin{array}{l}\text { High } \\
\text { confidence }\end{array}$ & $\begin{array}{l}\text { Minor concerns regarding methodological } \\
\text { limitations, no or very minor concerns } \\
\text { regarding coherence, adequacy, and } \\
\text { relevance }\end{array}$ \\
\hline & $\begin{array}{l}\text { Conflict with clinical autonomy: guideline } \\
\text { recommendations conflict with health } \\
\text { professional's independence for clinical } \\
\text { judgement }\end{array}$ & $\begin{array}{l}{[36,40,46,47} \\
49,51,53,56 \\
58-60,62]\end{array}$ & 13 & $\begin{array}{l}\text { High } \\
\text { confidence }\end{array}$ & $\begin{array}{l}\text { Minor concerns regarding methodological } \\
\text { limitations, no or very minor concerns } \\
\text { regarding coherence, adequacy, and } \\
\text { relevance }\end{array}$ \\
\hline & $\begin{array}{l}\text { Emotional responses to work and confidence } \\
\text { in skills: staff having lack of interest, } \\
\text { negative attitude towards work, or low } \\
\text { confidence in their ability to carry out } \\
\text { guideline recommendation }\end{array}$ & $\begin{array}{l}{[37,40,51,56} \\
57,59,61,62 \\
65]\end{array}$ & 12 & $\begin{array}{l}\text { Moderate } \\
\text { confidence }\end{array}$ & $\begin{array}{l}\text { Moderate concerns regarding } \\
\text { methodological limitations, minor } \\
\text { concerns regarding adequacy, and no or } \\
\text { very minor concerns regarding coherence } \\
\text { and relevance }\end{array}$ \\
\hline & $\begin{array}{l}\text { Competing priorities: staff burdened with } \\
\text { too many tasks to place guideline } \\
\text { adherence at high priority }\end{array}$ & $\begin{array}{l}{[36,38,44,46} \\
50,52,56,57 \\
60,63,67]\end{array}$ & 12 & $\begin{array}{l}\text { High } \\
\text { confidence }\end{array}$ & $\begin{array}{l}\text { Minor concerns regarding methodological } \\
\text { limitations, no or very minor concerns } \\
\text { regarding coherence, adequacy, and } \\
\text { relevance }\end{array}$ \\
\hline & $\begin{array}{l}\text { Reluctance to change: comfort with existing } \\
\text { behaviour and resistance to developing } \\
\text { new ones. }\end{array}$ & $\begin{array}{l}{[37,38,40,41} \\
43-46,51,52 \\
54,65]\end{array}$ & 11 & $\begin{array}{l}\text { High } \\
\text { confidence }\end{array}$ & $\begin{array}{l}\text { Minor concerns regarding methodological } \\
\text { limitations, no or very minor concerns } \\
\text { regarding coherence, adequacy, and } \\
\text { relevance }\end{array}$ \\
\hline & $\begin{array}{l}\text { Inconsistent practices: variations in practice } \\
\text { between different health professionals in }\end{array}$ & $\begin{array}{l}{[45,46,49,56,} \\
57]\end{array}$ & 8 & $\begin{array}{l}\text { Moderate } \\
\text { confidence }\end{array}$ & $\begin{array}{l}\text { Moderate concerns regarding } \\
\text { methodological limitations, minor }\end{array}$ \\
\hline
\end{tabular}


Table 4 GRADE-CERQual summary of qualitative review findings table: barriers and facilitators of implementing evidence-based guidelines in long-term care (Continued)

\begin{tabular}{lll} 
Summary of review finding & $\begin{array}{l}\text { Contributing } \\
\text { articles }\end{array}$ & $\begin{array}{l}\text { Frequency } \\
\text { CERQual } \\
\text { Assessment of } \\
\text { confidence in } \\
\text { the evidence }\end{array}$ \\
\hline
\end{tabular}

the LTC homes.

Moral distress: guideline conflicts with resident/staff values or generate perception 56$]$ that the guideline will cause negative outcomes.

Guideline complexity and associated workload: guideline creates additional workload to the staff due to the nature of its recommendations or complexity to process and understand the tasks

Healthcare system structure: inability to follow the guidelines due to the organizational structure of the healthcare system

Simultaneous changes or change fatigue: guideline introduces too many changes at once or staff are burdened with too many changes

Limited physical environment: lack of appropriate physical infrastructure to carry out guideline recommendations

Conflicting guidelines: guideline conflicts with another guideline on the same topic or current practice in the LTC homes

Impractical guideline: guideline is not practical to the LTC setting

Reactive approach: responding to problems once they occur rather than focusing on prevention

Lack of noticeable improvement from quideline implementation

Leadership and champions: LTC managers and leaders support the guideline implementation. Experienced champions are present to actively promote change and provide support to organizational members

Well-designed strategies, protocols, and resources: designing strategies, protocols, and tools that promote guideline uptake and minimize burden on the LTC system

Facilitators Support and coordination among staff: collaborative decision-making, clear role coordination, and encouragement among LTC staff

Adequate knowledge and education:

$[36,41,48,53,8$

$$
56]
$$

$[36,39,41,46,8$ $56,65]$

$[36,51,54,56] 5$

$[37,53,54]$

$[39,47,56,61] \quad 4$

$[36,47,56]$

$[44,60]$

2

$[36,48]$

2

$[65,67]$

2

$[28,37,40,44,20$ $46,49,52-54$,

$57,64,65]$

$[28,38,40,44,19$

$53,55,57,64$,

$65,67]$

$[28,37,42,44,18$ $49,50,57,61$

$64,65,67]$

$[37,38,46,50,16$
Moderate confidence

Explanation of CERQual assessment

concerns regarding adequacy, and no or very minor concerns regarding coherence and relevance

Moderate concerns regarding methodological limitations, minor concerns regarding adequacy, and no or very minor concerns regarding coherence and relevance

Moderate confidence

Moderate concerns regarding methodological limitations, minor concerns regarding adequacy, and no or very minor concerns regarding coherence and relevance

Moderate confidence

Moderate concerns regarding methodological limitations, minor concerns regarding adequacy, and no or very minor concerns regarding coherence and relevance

Moderate confidence

Moderate concerns regarding adequacy, minor concerns regarding methodological limitations, and no or very minor concerns regarding coherence and relevance

Moderate confidence

Moderate concerns regarding methodological limitations and adequacy, no or very minor concerns regarding coherence and relevance

Moderate confidence

Moderate concerns regarding adequacy, minor concerns regarding methodological limitations, and no or very minor concerns regarding coherence and relevance

High confidence, moderate confidence

Minor concerns regarding methodological limitations and adequacy, no or very minor concerns regarding coherence and relevance

Moderate concerns regarding adequacy,

Moderate confidence, high confidence minor concerns regarding methodological limitations, and no or very minor concerns regarding coherence and relevance

Moderate confidence

Moderate concerns regarding adequacy, minor concerns regarding methodological limitations, and no or very minor concerns regarding coherence and relevance

High Minor concerns regarding methodological confidence limitations, no or very minor concerns regarding coherence, adequacy, and relevance

High confidence

Minor concerns regarding methodological imitations, no or very minor concerns regarding coherence, adequacy, and relevance

High confidence Minor concerns regarding methodological limitations, no or very minor concerns regarding coherence, adequacy, and relevance

Minor concerns regarding methodological 
Table 4 GRADE-CERQual summary of qualitative review findings table: barriers and facilitators of implementing evidence-based guidelines in long-term care (Continued)

\begin{tabular}{|c|c|c|c|c|}
\hline Summary of review finding & $\begin{array}{l}\text { Contributing } \\
\text { articles }\end{array}$ & Frequency & $\begin{array}{l}\text { CERQual } \\
\text { Assessment of } \\
\text { confidence in } \\
\text { the evidence }\end{array}$ & Explanation of CERQual assessment \\
\hline $\begin{array}{l}\text { continuous education and training specific } \\
\text { to the LTC context to ensure that the care } \\
\text { team have the knowledge and skills to } \\
\text { carry out guideline interventions }\end{array}$ & $\begin{array}{l}52,55,57-59 \\
61,63-65,67]\end{array}$ & & confidence & $\begin{array}{l}\text { limitations, no or very minor concerns } \\
\text { regarding coherence, adequacy, and } \\
\text { relevance }\end{array}$ \\
\hline $\begin{array}{l}\text { Involving residents and families: engaging } \\
\text { residents and families in decision-making } \\
\text { and education }\end{array}$ & $\begin{array}{l}{[38,42,44,50} \\
53,57,63,65]\end{array}$ & 13 & $\begin{array}{l}\text { High } \\
\text { confidence, } \\
\text { high } \\
\text { confidence }\end{array}$ & $\begin{array}{l}\text { Minor concerns regarding methodological } \\
\text { limitations, no or very minor concerns } \\
\text { regarding coherence, adequacy, and } \\
\text { relevance }\end{array}$ \\
\hline $\begin{array}{l}\text { Positive emotional responses to work and the } \\
\text { intervention: the resident's care team value } \\
\text { the intervention and demonstrate interest } \\
\text { in developing care }\end{array}$ & $\begin{array}{l}{[40,52-54,64} \\
65]\end{array}$ & 13 & $\begin{array}{l}\text { High } \\
\text { confidence }\end{array}$ & $\begin{array}{l}\text { Minor concerns regarding methodological } \\
\text { limitations, no or very minor concerns } \\
\text { regarding coherence, adequacy, and } \\
\text { relevance }\end{array}$ \\
\hline $\begin{array}{l}\text { Adequate services, resources, and time: staff } \\
\text { have enough resources and time to carry } \\
\text { out guideline interventions }\end{array}$ & $\begin{array}{l}{[28,44,46,49} \\
50,54,57,64]\end{array}$ & 12 & $\begin{array}{l}\text { High } \\
\text { confidence }\end{array}$ & $\begin{array}{l}\text { Minor concerns regarding methodological } \\
\text { limitations, no or very minor concerns } \\
\text { regarding coherence, adequacy, and } \\
\text { relevance }\end{array}$ \\
\hline $\begin{array}{l}\text { Noticeable outcomes from guideline } \\
\text { implementation: positive outcomes } \\
\text { following guideline usage }\end{array}$ & $\begin{array}{l}{[28,37,44,47} \\
53,64]\end{array}$ & 12 & $\begin{array}{l}\text { Moderate } \\
\text { confidence }\end{array}$ & $\begin{array}{l}\text { Moderate concerns regarding } \\
\text { methodological limitations, minor } \\
\text { concerns regarding adequacy, and no or } \\
\text { very minor concerns regarding coherence } \\
\text { and relevance }\end{array}$ \\
\hline $\begin{array}{l}\text { Good communication and information flow: } \\
\text { information regarding new protocols or } \\
\text { resident assessment is communicated } \\
\text { promptly and regularly to and among the } \\
\text { resident's care team }\end{array}$ & $\begin{array}{l}{[42,44,50,54} \\
55]\end{array}$ & 7 & $\begin{array}{l}\text { Moderate } \\
\text { confidence }\end{array}$ & $\begin{array}{l}\text { Moderate concerns regarding } \\
\text { methodological limitations, no or very } \\
\text { minor concerns regarding coherence, } \\
\text { adequacy, and relevance }\end{array}$ \\
\hline $\begin{array}{l}\text { Conviction that the guideline is evidence- } \\
\text { based and will demonstrate improvement: } \\
\text { the resident's care team believe that the } \\
\text { guideline is evidence-based and that } \\
\text { guideline interventions will lead to positive } \\
\text { outcomes }\end{array}$ & {$[44,50]$} & 5 & Low confidence & $\begin{array}{l}\text { Serious concerns regarding adequacy, } \\
\text { minor concerns regarding methodological } \\
\text { limitations, no or very minor concerns } \\
\text { regarding coherence and relevance }\end{array}$ \\
\hline $\begin{array}{l}\text { Innovative environmental modifications: } \\
\text { innovative physical modification in the } \\
\text { physical environment that promotes } \\
\text { guideline usage }\end{array}$ & {$[38,63,67]$} & 5 & $\begin{array}{l}\text { High } \\
\text { confidence }\end{array}$ & $\begin{array}{l}\text { Minor concerns regarding methodological } \\
\text { limitations, no or very minor concerns } \\
\text { regarding coherence, adequacy, and } \\
\text { relevance }\end{array}$ \\
\hline
\end{tabular}

\section{Discussions}

\section{Summary of the main findings}

We systematically identified barriers and facilitators to implementing evidence-based guidelines in LTC and used behaviour change theory to link them to candidate knowledge translation functions. Across several guideline topics, time constraints and inadequate staffing, cost and lack of resources, knowledge gaps, and lack of teamwork and organizational support were frequently identified barriers. In contrast, leadership and champions, well-designed strategies, protocols, and resources, and adequate services, resources and time were frequently identified as facilitators. Linking to the central components of the Behaviour Change Wheel suggests physical and social opportunities and psychological capability are common targets for change to overcome barriers and leverage facilitators. While the most frequently identified barriers and facilitators appear to be universal regardless of guideline topics (e.g., pain, mood, physical activity, heart failure), some guidelines may have nuanced actions that have unique barriers and facilitators. We suggest that future knowledge translation and implementation science researchers assume the most frequently identified barriers and facilitators in our review are present and that they design strategies targeted at physical and social opportunity and psychological capability. A further analysis of barriers and facilitators may be necessary if the actions outlined by the guideline have unique features that could create additional barriers and facilitators.

The reported barriers and facilitators in our qualitative systematic review most frequently mapped onto the central Behaviour Change Wheel components physical and social opportunity: the opportunities afforded by the 
Table 5 Barrier and facilitator themes linked to COM-B constructs and Behaviour Change Wheel intervention functions

COM-B construct
Physical capability: physical skill, strength, or stamina
Psychological capability: knowledge or psychological skills,
strength or stamina to engage in the necessary mental processes

Physical opportunity: opportunity afforded by the environment involving time, resources, locations, cues, physical affordance

Social opportunity: opportunity afforded by the interpersonal influences, social cues and cultural norms that influence the way that we think about things

Reflective motivation: reflective processes involving plans (selfconscious intentions) and evaluations (beliefs about what is good and bad)

Automatic motivation: automatic processes involving emotional reactions, desires (wants and needs), impulses, inhibitions, drive states and reflex responses

\begin{tabular}{|c|c|}
\hline & Theme \\
\hline & None \\
\hline Barriers & Knowledge gaps \\
\hline \multirow[t]{2}{*}{ Facilitators } & Adequate knowledge and education \\
\hline & $\begin{array}{l}\text { Time constraints and inadequate } \\
\text { staffing }\end{array}$ \\
\hline \multirow[t]{10}{*}{ Barriers } & Cost and lack of resources \\
\hline & Resident complexity \\
\hline & $\begin{array}{l}\text { Compromised communication and } \\
\text { information flow }\end{array}$ \\
\hline & Staff turnover \\
\hline & Competing priorities \\
\hline & $\begin{array}{l}\text { Guideline complexity and associated } \\
\text { workload }\end{array}$ \\
\hline & Healthcare system structure \\
\hline & Limited physical environment \\
\hline & Conflicting guidelines \\
\hline & Impractical guideline \\
\hline \multirow[t]{3}{*}{ Facilitators } & $\begin{array}{l}\text { Well-designed strategies, protocols, } \\
\text { and resources }\end{array}$ \\
\hline & $\begin{array}{l}\text { Adequate services, resources, and } \\
\text { time }\end{array}$ \\
\hline & $\begin{array}{l}\text { Innovative environmental } \\
\text { modifications }\end{array}$ \\
\hline
\end{tabular}

Barriers

Lack of teamwork

Lack of organizational support

Inconsistent practices

Reactive approach

Facilitators Leadership and champions

Support and coordination among

$$
\text { staff }
$$

Involving residents and families

Good communication and

information flow

Barriers

Conflict with clinical autonomy

Belief against the guideline

Moral distress

Lack of noticeable outcomes from guideline implementation

Facilitators Noticeable outcomes from guideline implementation

Conviction that the guideline is evidence-based and will demonstrate improvement

Barriers Reluctance to change

Emotional responses to work and confidence in skills
Behaviour Change Whee

linked potential

intervention functions

None

Education

Training

Environmental restructuring

Modelling

Enablement

Training

Restriction

Environmental restructuring

Enablement

Restriction

Environmental restructuring

Modelling

Enablement

Education

Persuasion

Modelling

Enablement

Incentivisation

Coercion

Training

Incentivisation

Coercion

Environmental restructuring 
Table 5 Barrier and facilitator themes linked to COM-B constructs and Behaviour Change Wheel intervention functions (Continued)

\begin{tabular}{lll}
\hline COM-B construct & Theme & $\begin{array}{l}\text { Behaviour Change Wheel } \\
\text { linked potential } \\
\text { intervention functions }\end{array}$ \\
\hline & $\begin{array}{l}\text { Simultaneous changes or change } \\
\text { fatigue }\end{array}$ & $\begin{array}{l}\text { Persuasion } \\
\text { Modelling } \\
\text { Enablement }\end{array}$ \\
& Facilitators & $\begin{array}{l}\text { Positive emotional responses to } \\
\text { work and the intervention }\end{array}$ \\
\hline
\end{tabular}

environment (e.g., time, resources, locations, cues, physical affordances) and interpersonal influences (e.g., social cues and cultural norms that influence the way we think about things). The findings that environmental opportunities (e.g., changing the social and physical context of care provision) are significant barriers to implementing evidence-based guidelines echo recent concerns surrounding quality of care provided in LTC highlighted by the COVID-19 pandemic [21] and is consistent with previous literature. Indeed, there have been recurrent reports of lack of funding and subsequent personnel shortages leading to decreased time to provide services to increasingly complex residents in LTC $[22,23]$. Limited teamwork has also previously been identified as a barrier in LTC [24]. Linkage within the Behaviour Change Wheel suggests that training, restriction, environmental restructuring, enablement, and modelling are candidate knowledge translation intervention functions to overcome the identified barriers and leverage the facilitators.

Given the recent international interest in improving LTC during and after the COVID-19 pandemic and the subsequent impetus to support significant changes to the sector [21, 25], several of the Behaviour Change Wheel identified intervention functions could be feasible. For example, environmental restructuring involves changing the physical or social context to support guideline implementation. Resident-centred care approaches restructure the environment of care provision around the resident and address several of the barriers and facilitators identified in our review. For example, one such evidence-based approach, Neighbourhood Team Development, focuses on modifying the physical LTC environment, reorganizing delivery of care services, and aligning team members (e.g., LTC staff, family, residents) to collaborate in providing care [26]. Several of the studies included in our review also identified involving residents and family members as a facilitator of implementing evidence-based guidelines, supporting a resident-centred care approach.

Knowledge gaps pertaining to the information within guidelines, change fatigue, and lack of interest in work were frequently identified barriers and facilitators in our systematic review, which mapped onto the COM-B domains of psychological capability and reflective and automatic motivation. In many countries, most direct care within LTC homes is provided by care aides (e.g., personal support workers, health care aides, continuing care assistants, resident assistants) [27, 28] who often have the lowest level of education, receive the lowest financial compensation, have the least autonomy, and experience work-related burnout and poor job satisfaction $[27,29]$. Knowledge gaps also apply to other members of the LTC interprofessional teams including licensed nurses, physicians, pharmacists, and rehabilitation and recreation and leisure providers. Indeed, several of the studies included in our review revealed knowledge gaps for different members of the LTC team. Education and training are potential knowledge translation intervention functions to overcome barriers associated with psychological capability and reflective and automatic motivation. Training for care aides is variable within and between countries. For example, in Canada, there are currently no national education standards for care aides working in LTC, and training varies widely between provinces [30]. Training of other members of the interprofessional team (e.g., physicians, physical therapists) often does not include a focus on geriatrics or LTC, nor is it standardized. Indeed, the COVID-19 pandemic revealed a major gap in standardized training for all team members about proper personal protective equipment use and conservation [31]. Consistent education and training with monitored national standards for all LTC staff may be one targeted knowledge translation strategy. However, for continuing education to be effective in LTC, it must be supported by the organization, and ongoing expert support is needed to enable and reinforce learning [32] which further bolsters the argument for a team-based, resident-centred approach.

\section{Comparison with other reviews and implications for the field}

This is the first study to synthesize barriers and facilitators to guideline implementation in LTC from the perspectives of staff across healthcare conditions. Barriers and facilitators to guideline implementation have been systematically reviewed in other healthcare settings, but 
until now, no syntheses have been developed for the LTC context. Further, we not only identified the barriers and facilitators but also mapped them onto the central constructs of the Behaviour Change Wheel. This helps us explore the reasons why the factors identified are barriers and facilitators and the findings can be used to inform the development of future theory-guided knowledge translation intervention development.

\section{Overall completeness and applicability of the evidence}

From a methodological point of view, the studies included in our review had several limitations. First, studies often did not report important information about the LTC home(s) which provides context from which the results were derived, such as the size, ownership, and rurality of the LTC home. The context of the LTC home including number of residents in a home, funding structure, and access to resources has been previously shown to affect implementation of best practice guidelines in LTC [14]. Future authors of LTC research are encouraged to fully describe the setting so that readers can adequately assess the generalizability of the results to their context, or reasons why they may experience different outcomes. Further, authors should include a fulsome description of the context including care philosophy of the home, staffing levels, and health system influences (e.g., public or private funding). Second, most authors did not critically examine their own role, potential bias, and influence during analysis and presentation of results. Reflexivity, or the acknowledgement of underlying beliefs and values held by researcher in selecting and justifying their methodological approach [33], is essential in assessing the authenticity of qualitative results [34]. Authors of qualitative research are encouraged to include a reflexive statement when reporting their results that describes their role in data collection, analysis and interpretation, and potential resulting biases that may arise.

\section{Limitations of the review}

A strength of our study is that we synthesized information across different health conditions within the LTC sector. Given that there are likely many similarities among barriers and facilitators across guidelines for different conditions in the LTC setting, the findings of this qualitative evidence synthesis can help inform the implementation of any evidence-based guideline in LTC homes. However, a limitation of our study is that we did not assess the strength of the barriers and facilitators identified in this review. A frequently identified barrier may not hinder implementation as much as one that is less frequently reported. We argue that frequently reported barriers across several guideline topics are nonetheless important to identify as they can inform design of knowledge translation strategies regardless of topic.
Future work should examine the strength of barriers and facilitators in LTC for implementing evidence-based guidelines and determine which barriers significantly limit implementation to add to our work. Another limitation is that we did not complete the third stage of the Thomas and Harden approach to thematic synthesis [19] to develop analytical themes that enable the development of new theoretical insights and findings not seen at individual primary study level. However, we saw mapping the barriers and facilitator themes onto the COM-B components as a way to take our analysis to the next step and provide recommendations for theory-guided knowledge translation strategies and understand why barriers and facilitators may exist. Additionally, as per the Thomas and Harden approach, we did not code directly onto any part of the manuscripts and focused our extraction on the results and findings sections, meaning key evidence may have been missed. We only included studies published in English which limits the generalizability of our findings to English-speaking countries or those that can pay for translation services. There is subjectivity in mapping of barriers and facilitators onto the COM-B components; some barriers and facilitators could map onto different components depending on the readers' interpretations. Though we identified candidate intervention functions for implementing guidelines in LTC, we did not assess which ones are feasible and realistic to implement. Our next steps are to use the APEASE criteria [35] in consultation with stakeholders to determine the most appropriate intervention functions for the LTC sector.

\section{Conclusion and implications Implications for practice}

We suggest that people designing LTC interventions to support guideline implementation assume the most frequently identified barriers (time constraints and inadequate staffing, cost and lack of resources, knowledge gaps, and lack of teamwork and organizational support) and facilitators (leadership and champions, welldesigned strategies, protocols, and resources, and adequate services, resources and time) in our review are present and design strategies targeted at physical and social opportunity and psychological capability. Further analysis of barriers and facilitators specific to the guideline they are implementing may be necessary if the actions outlined by the guideline have unique features that could cause additional barriers and facilitators.

\section{Implications for research}

Implications for research have been developed based on the findings of our study and our GRADE-CERQual assessment of findings. Future qualitative work in this area should transparently report researcher reflexivity 
including a reflection of the researchers' roles and the influence this may have on the findings of the study. Additionally, researchers must fully describe the context of their LTC setting to ensure readers can determine whether the findings apply to their local LTC context. A full description of context would include the care philosophy of the home, staffing levels, and health system influences (e.g., public or private funding) among other factors.

\section{Abbreviations}

COM-B: Capabilitiy, opportunity, motivation, behaviour; LTC: Long-term care

\section{Supplementary Information}

The online version contains supplementary material available at https://doi. org/10.1186/s13012-021-01140-0.
Additional file 1. ENTREQ Checklist.
Additional file 2. Search Strategy.
Additional file 3. Evidence Profile.
Additional file 4. CASP Checklist.
Additional file 5. Barriers and Facilitators Analysis.

\section{Acknowledgements}

When preparing this protocol/review, we used EPOC's Protocol and Review Template for Qualitative Evidence Synthesis (Glenton C, Bohren MA, Downe S, Paulsen EJ, Lewin S, on behalf of Effective Practice and Organisation of Care (EPOC). EPOC Qualitative Evidence Synthesis: Protocol and review template. Version 1.1. EPOC Resources for review authors. Oslo: Norwegian Institute of Public Health; 2020), available at: http://epoc.cochrane.org/epocspecific-resources-review-authors).

\section{Authors' contributions}

CM conceptualized the study; CM and YB conducted data analysis; CM, YB, and $\mathrm{PH}$ interpreted the findings; and $\mathrm{CM}$ and $\mathrm{YB}$ wrote the manuscript. LG, SS, and AP provided content expertise and assisted with interpretation of the findings. The authors critically read, contributed to, and approved the manuscript for submission.

\section{Funding}

CM was supported by a fellowship from the Canadian Institutes of Health Research. YB was supported by a summer studentship from the McMaster Institute for Research on Aging. The funder had no role in the study, interpretation of data, or decision to submit results.

\section{Availability of data and materials}

Not applicable.

\section{Declarations}

Ethics approval and consent to participate

Not applicable.

\section{Consent for publication}

Not applicable.

\section{Competing interests}

The authors declare no competing interests.

\section{Author details}

'Dalhousie University, 5869 University Avenue, Halifax, Nova Scotia B3H 4R2, Canada. ${ }^{2}$ GERAS Centre for Aging Research, 88 Maplewood Avenue, Hamilton, Ontario L8M 1W9, Canada. ${ }^{3}$ Master University, 1280 Main Street West, Hamilton, Ontario L8S 4L8, Canada. ${ }^{4}$ University of Waterloo, 200
University Avenue West, Waterloo, Ontario N2L 3G1, Canada. ${ }^{5}$ Schlegel-UW Research Institute for Aging, 150 Laurelwood Drive, Waterloo, Ontario N2J 0E2, Canada. ${ }^{6}$ University of Toronto, 27 King's College Circle, Toronto, Ontario M5S 1A4, Canada.

Received: 10 February 2021 Accepted: 24 June 2021

Published online: 09 July 2021

\section{References}

1. Davidoff F, Case K, Fried PW. Evidence-based medicine: why all the fuss? Ann Intern Med. 1995:122(9):727.

2. Bauer MS. A review of quantitative studies of adherence to mental health clinical practice guidelines. Harv Rev Psychiatry. 2002;10(3):138-53.

3. Mickan S, Burls A, Glasziou P. Patterns of "leakage" in the utilisation of clinical guidelines: a systematic review. Postgrad Med J. 2011;87(1032):6709.

4. Eccles M, Grimshaw J, Walker A, Johnston M, Pitts N. Changing the behavior of healthcare professionals: the use of theory in promoting the uptake of research findings. J Clin Epidemiol. 2005;58(2):107-12.

5. Grimshaw JM, Thomas RE, MacLennan G, Fraser C, Ramsay CR, Vale L, et al. Effectiveness and efficiency of guideline dissemination and implementation strategies. Health Technol Assess. 2004;8(6):1-72.

6. Helfrich CD, Damschroder LJ, Hagedorn HJ, Daggett GS, Sahay A, Ritchie M, et al. A critical synthesis of literature on the promoting action on research implementation in health services (PARIHS) framework. Implement Sci. 2010; 5(1):82.

7. Michie S, van Stralen MM, West R. The behaviour change wheel: a new method for characterising and designing behaviour change interventions. Implement Sci. 2011;6(1):42.

8. Wilson KM, Brady TJ, Lesesne C, NCCDPHP Work Group on Translation. An organizing framework for translation in public health: the knowledge to action framework - PubMed. Prev Chronic Dis. 2011;8(2):A46.

9. Ellen ME, Panisset U, Araujo de Carvalho I, Goodwin J, Beard J. A Knowledge Translation framework on ageing and health. Health Policy. 2017;121(3):282291.

10. U.S. Department of Health and Human Services. Why population aging matters - a global perspective. National Institute on Aging, National Institutes of Health; 2017

11. World Health Organization. The Global strategy and action plan on ageing and health. 2020. Available from: https://www.who.int/ageing/global-stra tegy/en/

12. Registered Nurses' Association of Ontario. Clinical BPGs. Long-term care best practices toolkit, 2nd edition. Available from: https://ltctoolkit.rnao.ca/clinica I-topics/bpgs. [cited 2020 Mar 23]

13. Bostrom A-M, Slaughter SE, Chojecki D, Estabrooks CA. What do we know about knowledge translation in the care of older adults? A scoping review. J Am Med Dir Assoc. 2012;13(3):210-9.

14. Estabrooks CA, Squires JE, Hayduk L, Morgan D, Cummings GG, Ginsburg L, et al. The influence of organizational context on best practice use by care aides in residential long-term care settings. J Am Med Dir Assoc. 2015;16(6): 537.e1-537.e10

15. Francke AL, Smit MC, De Veer AJE, Mistiaen P. Factors influencing the implementation of clinical guidelines for health care professionals: a systematic meta-review. BMC Med Inform Decis Mak. 2008:8:38.

16. Noyes J, Booth A, Cargo M, Flemming K, Garside R, Hannes K, et al. Cochrane Qualitative and Implementation Methods Group guidance seriespaper 1: introduction. J Clin Epidemiol. 2018;97:35-8.

17. Tong A, Flemming K, Mclnnes E, Oliver S, Craig J. Enhancing transparency in reporting the synthesis of qualitative research: ENTREQ. BMC Med Res Methodol. 2012;12(1):181.

18. CASP. Qualitative appraisal checklist for qualitative research. Critical Appraisal Skills Programme. 2011. Available from: https://casp-uk.net/casptools-checklists/. [cited 2020 May 28]

19. Thomas J, Harden A. Methods for the thematic synthesis of qualitative research in systematic reviews. BMC Med Res Methodol. 2008:8:45.

20. Lewin S, Booth A, Glenton C, Munthe-Kaas H, Rashidian A, Wainwright M, et al. Applying GRADE-CERQual to qualitative evidence synthesis findings: introduction to the series. Implement Sci. 2018;13(Suppl 1):2.

21. Grabowski DC, Mor V. Nursing home care in crisis in the wake of COVID-19. JAMA. 2020;324(1):23-4. 
22. Castle NG, Engberg J. Staff turnover and quality of care in nursing homes. Med Care. 2005;43(6):616-26.

23. Ng R, Lane N, Tanuseputro P, Mojaverian N, Talarico R, Wodchis WP, et al. Increasing complexity of new nursing home residents in Ontario, Canada: a serial cross-sectional study. J Am Geriatr Soc. 2020.

24. Kane R, West J. It shouldn't be this way: the failure of long-term care. Nashville: Vanderbilt University Press; 2005.

25. Gordon A, Goodman C, Achterberg W, Barker RO, Burns E, Hanratty B, et al. Commentary: COVID in care homes-challenges and dilemmas in healthcare delivery - PubMed. Age Ageing. 2020;13:afaa113.

26. Boscart VM, Sidani S, Ploeg J, Dupuis SL, Heckman G, Kim JL, et al. Neighbourhood Team Development to promote resident centred approaches in nursing homes: a protocol for a multi component intervention. BMC Health Serv Res. 2019;19(1):922.

27. Andersen E. Working in long-term residential care: a qualitative meta summary encompassing roles, working environments, work satisfaction, and factors affecting recruitment and retention of nurse aides. Glob J Health Sci. 2009;1(2):2.

28. Berta W, Ginsburg L, Gilbart E, Lemieux-Charles L, Davis D. What, why, and how care protocols are implemented in Ontario nursing homes. Can J Aging Rev Can Vieil. 2013;32(1):73-85.

29. Caspar S, Ratner PA, Phinney A, MacKinnon K. The influence of organizational systems on information exchange in long-term care facilities. Qual Health Res. 2016;26(7):951-65.

30. Association of Canadian Community Colleges. Canadian educational standards for personal care providers. 2012.

31. Kimball A, Hatfield KM, Arons M, James A, Taylor J, Spicer K, et al. Asymptomatic and presymptomatic SARS-COV-2 infections in residents of a long-term care skilled nursing facility - King County, Washington, March 2020. Morb Mortal Wkly Rep. 2020;69(13):377-81.

32. Stolee P, Esbaugh J, Aylward S, Cathers T, Harvey DP, Hillier LM, et al. Factors associated with the effectiveness of continuing education in longterm care. Gerontologist. 2005;45(3):399-409.

33. Shacklock G, Smyth J. Being reflexive in critical educational and social research. London: Falmer; 1998.

34. Reid AM, Brown JM, Smith JM, Cope AC, Jamieson S. Ethical dilemmas and reflexivity in qualitative research. Perspect Med Educ. 2018;7(2):69-75.

35. Michie S West R. AL. The Behaviour Change Wheel: a guide to designing interventions. Silverback Publishing; 2014.

36. Phipps E, Watson C, Mearkle R, Lock S. Influenza in carehome residents: applying a conceptual framework to describe barriers to the implementation of guidance on treatment and prophylaxis. J Public Health Oxf Engl. 2020;43(3):602-9.

37. Abraham J, Kupfer R, Behncke A, Berger-Hoger B, Icks A, Haastert B, et al. Implementation of a multicomponent intervention to prevent physical restraints in nursing homes (IMPRINT): a pragmatic cluster randomized controlled trial. Int J Nurs Stud. 2019;96:27-34.

38. Villarosa AR, Clark S, Villarosa AC, Patterson Norrie T, Macdonald S, Anlezark $J$, et al. Promoting oral health care among people living in residential aged care facilities: perceptions of care staff. Gerodontology. 2018;35(3):177-84.

39. Huhtinen E, Quinn E, Hess I, Najjar Z, Gupta L. Understanding barriers to effective management of influenza outbreaks by residential aged care facilities. Australas J Ageing. 2019;38(1):60-3.

40. Nilsen P, Wallerstedt B, Behm L, Ahlstrom G. Towards evidence-based palliative care in nursing homes in Sweden: a qualitative study informed by the organizational readiness to change theory. Implement Sci IS. 2018;13(1):1.

41. DuBeau CE, Ouslander JG, Palmer MH. Knowledge and attitudes of nursing home staff and surveyors about the revised federal guidance for incontinence care. Gerontologist. 2007;47(4):468-79.

42. Birney A, Charland P, Cole M. Evaluation of the antipsychotic medication review process at four long-term facilities in Alberta. J Multidiscip Healthc. 2016;9:499-509.

43. Fallon T, Buikstra E, Cameron M, Hegney D, Mackenzie D, March J, et al. Implementation of oral health recommendations into two residential aged care facilities in a regional Australian city. Int J Evid Based Healthc. 2006;4(3): 162-79

44. Baert V, Gorus E, Calleeuw K, De Backer W, Bautmans I. An administrator's perspective on the organization of physical activity for older adults in longterm care facilities. J Am Med Dir Assoc. 2016;17(1):75-84.

45. Alamri SH, Kennedy CC, Marr S, Lohfeld L, Skidmore CJ, Papaioannou A. Strategies to overcome barriers to implementing osteoporosis and fracture prevention guidelines in long-term care: a qualitative analysis of action plans suggested by front line staff in Ontario, Canada. BMC Geriatr. 2015;15: 94.

46. Kaasalainen S, Ploeg J, Donald F, Coker E, Brazil K, Martin-Misener R, et al. Positioning clinical nurse specialists and nurse practitioners as change champions to implement a pain protocol in long-term care. Pain Manag Nurs Off J Am Soc Pain Manag Nurses. 2015;16(2):78-88.

47. Vikstrom S, Sandman P-O, Stenwall E, Bostrom A-M, Saarnio L, Kindblom K, et al. A model for implementing guidelines for person-centered care in a nursing home setting. Int Psychogeriatr. 2015;27(1):49-59.

48. Strachan $\mathrm{PH}$, Kaasalainen $\mathrm{S}$, Horton A, Jarman H, D'Elia T, Van Der Horst M-L, et al. Managing heart failure in the long-term care setting: nurses' experiences in Ontario, Canada. Nurs Res. 2014;63(5):357-65.

49. Lim CJ, Kwong M, Stuart RL, Buising KL, Friedman ND, Bennett N, et al. Antimicrobial stewardship in residential aged care facilities: need and readiness assessment. BMC Infect Dis. 2014;14:410.

50. Dellefield ME, Magnabosco JL. Pressure ulcer prevention in nursing homes: nurse descriptions of individual and organization level factors. Geriatr Nurs N Y N. 2014;35(2):97-104

51. Bamford C, Heaven B, May C, Moynihan P. Implementing nutrition guidelines for older people in residential care homes: a qualitative study using Normalization Process Theory. Implement Sci IS. 2012;7:106.

52. Kaasalainen S, Brazil K, Akhtar-Danesh N, Coker E, Ploeg J, Donald F, et al. The evaluation of an interdisciplinary pain protocol in long term care. J Am Med Dir Assoc. 2012;13(7):664.e1-8.

53. Verkaik R, Francke AL, van Meijel B, Ouwerkerk J, Ribbe MW, Bensing JM. Introducing a nursing guideline on depression in dementia: a multiple case study on influencing factors. Int J Nurs Stud. 2011;48(9):1129-39.

54. Berta W, Teare GF, Gilbart E, Ginsburg LS, Lemieux-Charles L, Davis D, et al. Spanning the know-do gap: understanding knowledge application and capacity in long-term care homes. Soc Sci Med. 2010;70(9):1326-34.

55. McConigley R, Toye C, Goucke R, Kristjanson LJ. Developing recommendations for implementing the Australian Pain Society's pain management strategies in residential aged care. Australas J Ageing. 2008; 27(1):45-9.

56. Cheek J, Gilbert A, Ballantyne A, Penhall R. Factors influencing the implementation of quality use of medicines in residential aged care. Drugs Aging. 2004;21(12):813-24.

57. Hilton S, Sheppard JJ, Hemsley B. Feasibility of implementing oral health guidelines in residential care settings: views of nursing staff and residential care workers. Appl Nurs Res. 2016;30:194-203.

58. Lau D, Banaszak-Holl J, Nigam A. Perception and use of guidelines and interprofessional dynamics: assessing their roles in guideline adherence in delivering medications in nursing homes. Qual Manag Health Care. 2007; 16(2):135-45.

59. Buss I, Halfens R, Abu-Saad H, Kok G. Pressure ulcer prevention in nursing homes: views and beliefs of enrolled nurses and other health care workers. J Clin Nurs Wiley-Blackwell. 2004;13(6):668-76.

60. Maaden T, Steen JT, Koopmans RTCM, Doncker SMMM, Anema JR, Hertogh CMPM, et al. Symptom relief in patients with pneumonia and dementia: implementation of a practice guideline. Int J Geriatr Psychiatry. 2017;32(8): 829-39.

61. Kong $\mathrm{E}-\mathrm{H}$, Kim H, Kim H. Nursing home staff's perceptions of barriers and needs in implementing person-centred care for people living with dementia: a qualitative study. J Clin Nurs. 2021;23. Epub ahead of print

62. Jeong E, Park J, Chang SO. Development and evaluation of clinical practice guideline for delirium in long-term care. Int J Environ Res Public Health. 2020;17(21):8255.

63. Eldh AC, Rycroft-Malone J, van der Zijpp T, McMullan C, Hawkes C. Using nonparticipant observation as a method to understand implementation context in evidence-based practice. Worldviews Evid Based Nurs. 2020;17(3): 185-92.

64. Cossette B, Bruneau M-A, Couturier Y, Gilbert S, Boyer D, Ricard J, et al. Optimizing Practices, Use, Care and Services-Antipsychotics (OPUS-AP) in long-term care centers in Québec, Canada: a strategy for best practices. J Am Med Dir Assoc. 2020;21(2):212-9.

65. Surr CA, Holloway I, Walwyn RE, Griffiths AW, Meads D, Kelley R, et al. Dementia Care Mapping ${ }^{T M}$ to reduce agitation in care home residents with dementia: the EPIC cluster RCT. Health Technol Assess Winch Engl. 2020; 24(16):1-172. 
66. Desveaux L, Halko R, Marani H, Feldman S, Ivers NM. Importance of team functioning as a target of quality improvement initiatives in nursing homes: a qualitative process evaluation. J Contin Educ Health Prof. 2019;39(1):21-8.

67. Walker P, Kifley A, Kurrle S, Cameron ID. Process outcomes of a multifaceted, interdisciplinary knowledge translation intervention in aged care: results from the vitamin D implementation (ViDAus) study. BMC Geriatr. 2019;19(1):177.

\section{Publisher's Note}

Springer Nature remains neutral with regard to jurisdictional claims in published maps and institutional affiliations.

Ready to submit your research? Choose BMC and benefit from:

- fast, convenient online submission

- thorough peer review by experienced researchers in your field

- rapid publication on acceptance

- support for research data, including large and complex data types

- gold Open Access which fosters wider collaboration and increased citations

- maximum visibility for your research: over $100 \mathrm{M}$ website views per year

At $B M C$, research is always in progress.

Learn more biomedcentral.com/submissions 